Pacific

Journal of

Mathematics

QUATERNIONIC REPRESENTATIONS OF EXCEPTIONAL LIE GROUPS

Hung Yean LoKe

Volume $211 \quad$ No. 2

October 2003 


\title{
QUATERNIONIC REPRESENTATIONS OF EXCEPTIONAL LIE GROUPS
}

\author{
HUNG YEAN LOKE
}

\begin{abstract}
Let $G$ be a quaternionic real form of an exceptional group of real rank 4. Gross and Wallach show that three representations in the continuation of the quaternionic discrete series are unitarizable (see Gross and Wallach, 1996). In this paper we will determine the restrictions of these representations to certain subgroups of $G$ by computing explicitly the intersections of orbits. In particular we will determine certain compact dual pair correspondences of the minimal representation of G.
\end{abstract}

\section{Introduction.}

1.1. We refer to $\S 3$ of $[\mathbf{G W 2}]$ or $\S 2$ of [L3] for the definition of the double cover $G$ of a quaternionic real form $G_{0}$ of a complex Lie group $G(\mathbb{C})$. $G$ has maximal compact subgroup $K$ of the form $M_{1} \times M$ where $M_{1}$ is isomorphic to $S U_{2}$. Its Lie algebra has complexified Cartan decomposition $\mathfrak{g}=\mathfrak{k} \oplus \mathfrak{p}$. Here $\mathfrak{p}=\mathbb{C}^{2} \otimes V_{M}$ where $V_{M}$ is a self dual representation of $M(\mathbb{C})$.

1.2. Let $G(\mathbb{C})$ be one of the following simply connected complex exceptional groups:

$$
F_{4}(\mathbb{C}), E_{6}(\mathbb{C}) \rtimes \mathbb{Z} / 2 \mathbb{Z}, E_{7}(\mathbb{C}), E_{8}(\mathbb{C}) .
$$

We will index these four cases by $s=1,2,4,8$ respectivley. Let $G(\mathbb{C})^{0}$ denote the connected component of $G(\mathbb{C})$. Then there exists a unique connected quaternionic real form $G_{0}^{0}$ of $G(\mathbb{C})^{0}$ (cf. $\S 1.1$ ). It has a real root system of type $F_{4}$. We will denote $G_{0}^{0}$ by $F_{4,4}, E_{6,4}, E_{7,4}$ and $E_{8,4}$ respectively. Set $G_{0}=F_{4,4}, E_{6,4} \rtimes \mathbb{Z} / 2 \mathbb{Z}, E_{7,4}$ and $E_{8,4}$ respectively and let $G$ denote the corresponding double cover of $G_{0}$.

It is known that $\mathbb{P} V_{M}$ is a union of four $M(\mathbb{C})$-orbits. One of the orbits is Zariski dense and we will follow $[$ GW2] and denote the Zariski closure of the remaining three orbits by $X, Y$ and $Z$. Here $Z$ is the unique closed orbit in $\mathbb{P} V_{M}$ and

$$
\mathbb{P} V_{M} \supset X \supset Y \supset Z \text {. }
$$

Let $\mathcal{O}$ be either $X, Y$ or $Z$ and let $\bigoplus_{n} A^{n}(\mathcal{O})$ denote the coordinate ring of $\mathcal{O}$ in $\mathbb{P} V_{M}$. Note that $A^{n}(\mathcal{O})$ is a representation of $M$. 
In [GW1], [GW2] Gross and Wallach construct a unitary representation $\sigma_{\mathcal{O}}$ in the continuations of the quaternionic discrete series which is associated to $\mathcal{O}$ in the sense that it has $K$-types $\left(K=S U_{2} \times M\right)$

$$
\sum_{n=0}^{\infty} \operatorname{Sym}^{n+k}\left(\mathbb{C}^{2}\right) \otimes A^{n}(\mathcal{O})
$$

where $k$ is the integer $3 s+3,2 s+2$ and $s+2$ if $\mathcal{O}$ is $X, Y$ and $Z$ respectively. Again we will follow the notations of [GW2] and denote the three representations by $\sigma_{X}, \sigma_{Y}$ and $\sigma_{Z}$.

1.3. Let $G$ be one of the exceptional groups of real rank 4 in $\S 1.2$. A quaternionic Lie subgroup $G^{\prime}$ of $G$ is defined as a semisimple Lie subgroup of $G$ containing $M_{1}$. Let $M^{\prime}=G^{\prime} \cap M$.

We refer to $\S 2$ and its references for the definition of a quaternionic representation of $G$ and $G^{\prime}$. In [L3] we show that the restriction of $\sigma_{\mathcal{O}}$ from $G$ to $G^{\prime}$ decomposes discretely into a direct sum of quaternionic representations of $G^{\prime}$. There we derived a formula to compute the spectrum of decompositions (See Thm. 3.4.1 [L3]) and we deduced that most of the irreducible components of the restriction to $G^{\prime}$ are determined by the coordinate ring of the intersection of $\mathcal{O} \cap \mathbb{P} V_{0}$. Here $\mathbb{P} V_{0}$ is a $M^{\prime}(\mathbb{C})$-invariant subspace of $\mathbb{P} V_{M}$ and hence $\mathcal{O} \cap \mathbb{P} V_{0}$ is a $M^{\prime}(\mathbb{C})$-invariant projective variety. We will briefly recall these results in $\S 3$. Unfortunately the formula cannot be applied immediately to some interesting situations. In particular the coordinate ring of $\mathcal{O} \cap \mathbb{P} V_{0}$ is in general difficult to compute.

This paper is a continuation of [L3]. The main objective of this paper is to determine the restriction of $\sigma_{\mathcal{O}}$ in the following five situations (cf. (8)):

$$
\begin{array}{rll}
G=\widetilde{F}_{4,4} & \supset & G^{\prime}=\widetilde{\operatorname{Spin}}(5,4) \times_{(\mathbb{Z} / 2 \mathbb{Z})^{2}}(\mathbb{Z} / 2 \mathbb{Z})^{2} \\
G=\widetilde{F}_{4,4} & \supset & G^{\prime}=\widetilde{\operatorname{Spin}}(4,4) \times_{(\mathbb{Z} / 2 \mathbb{Z})^{3}}(\mathbb{Z} / 2 \mathbb{Z})^{3} \\
G=\widetilde{E}_{6,4} \rtimes(\mathbb{Z} / 2 \mathbb{Z}) & \supset & G^{\prime}=\widetilde{F}_{4,4} \times(\mathbb{Z} / 2 \mathbb{Z}) \\
G=\widetilde{E}_{7,4} & \supset & G^{\prime}=\widetilde{E}_{6,4} \times_{\mu_{3}} U_{1} \\
G=\widetilde{E}_{8,4} & \supset & G^{\prime}=\widetilde{E}_{7,4} \times_{\mu_{2}} S U_{2} .
\end{array}
$$

Here the tilde above the group denotes its double cover. We will compute the decompositions of the restrictions of the representations $\sigma_{\mathcal{O}}$ by explicitly computing the intersections $\mathcal{O} \cap \mathbb{P} V_{0}$. We have mentioned that one of the difficulty is to determine the intersections and their coordinate rings. Fortunately in each of the above $G^{\prime}$ it is known that $\mathbb{P} V_{0}$ is a union of finitely many $M^{\prime}(\mathbb{C})$ orbits and the intersection $\mathcal{O} \cap \mathbb{P} V_{0}$ can therefore be effectively determined. We remark that the above subgroups $G^{\prime}$ are just a few such examples. Our method presented in this paper should be applicable to other subgroups $G^{\prime}$ where $M^{\prime}(\mathbb{C})$ exhibit a dense orbit in $\mathbb{P} V_{0}$. 
The restrictions provide an efficient method for finding many quaternionic representations of the exceptional quaternionic Lie groups which are unitarizable. Since $\sigma_{\mathcal{O}}$ has small Gelfand-Kirillov dimensions, the quaternionic representations obtained in the restriction would have small Gelfand-Kirillov dimensions too.

1.4. The representation $\sigma_{Z}$ is a ladder representation and it is annihilated precisely by the Joseph ideal and it is thus called the minimal representation of $G$. A pair of reductive subgroups $\mathrm{H}_{1} \times_{C} \mathrm{H}_{2}$ in $G$ is called a (reductive) dual pair if the centralizers of $H_{1}$ and $H_{2}$ in $G$ are $H_{2}$ and $H_{1}$ respectively. The dual pair is called compact if either $H_{1}$ or $H_{2}$ is compact. Note that $G^{\prime}$ in (3) to (7) are examples of compact dual pairs. In the appendix of [L3], we showed that certain correspondences of dual pairs in $\S 1.3$ exist and the second objective of this paper is to find the rest (if any) of the correspondences. We will also describe the dual pairs correspondences of $\operatorname{Spin}(4,4) \times_{\mu_{2}^{2}} U_{1}^{2}$ in $E_{6,4} \rtimes \mathbb{Z} / 2 \mathbb{Z}$ in Theorem 7.3.1 and $E_{6,4} \times S U_{3}$ in $E_{8,4}$ in Corollary 4.10.1.

We remark that the restrictions of the minimal representations to dual pairs are of great interest. Exceptional dual pairs correspondences have been investigated by $[\mathbf{H P S}],[\mathbf{L i 1}],[\mathbf{L i 2}],[\mathbf{G S}]$ and $[\mathbf{L 2}]$. In $[\mathbf{G S}]$ and $[\mathbf{M S}]$, the authors computed certain dual pair correspondences of $p$-adic exceptional groups by determining the intersection of orbits.

1.5. The organization of the paper is as follows: In $\S 2$ we will recall the definition of quaternionic groups and quaternionic representations. In $\S 3$ we will briefly state the restriction formula of [L3]. The main results of this paper are stated in $\S 4$. In $\S 5$ we describe the closures of the orbits $X, Y, Z$ in detail. The rest of the paper is devoted to the proofs of the main results in $\S 4$.

1.6. We define some notations. $\pi_{G}\left(a_{1} \varpi_{1}+\cdots+a_{n} \varpi_{n}\right)$ will denote the irreducible finite dimensional complex representation of a semisimple Lie group $G$ with highest weight $a_{1} \varpi_{1}+\cdots+a_{n} \varpi_{n}$ where $\varpi_{i}$ are the fundamental weights given in Planches [Bou]. If $V$ is a representation of $G$, then $S^{n}(V)=\operatorname{Sym}^{n} V$ will denote its $n$-th symmetric product and $V^{*}$ its dual representation. $S^{n}$ will denote the representation $\operatorname{Sym}^{n}\left(\mathbb{C}^{2}\right)$ of $S U_{2} \cdot \chi_{1}$ will denote a fundamental character of the compact torus $U_{1} \cdot \mu_{n}$ will denote the cyclic group of order $n$. Suppose $H_{1}$ and $H_{2}$ are subgroups of $G$ and $C$ lies in the centers of both $H_{1}$ and $H_{2}$, then we denote

$$
H_{1} \times{ }_{C} H_{2}:=\left(H_{1} \times H_{2}\right) /\{(z, z): z \in C\} .
$$

\section{Quaternionic groups and representations.}

2.1. In this section we define some notations and briefly recall the definitions of the quaternionic real form of an algebraic group and quaternionic representations. 
2.2. We refer to $\S 3$ of $[\mathbf{G W 2}]$ and $\S 2$ of $[\mathbf{L 3}]$ for the definition of the quaternionic real form $\mathfrak{g}_{0}$ of a complex simple Lie algebra $\mathfrak{g}$. It has Cartan decomposition $\mathfrak{g}=\mathfrak{k} \oplus \mathfrak{p}$ where

$$
\mathfrak{k}=\mathfrak{s u}_{2} \oplus \mathfrak{m} .
$$

Here $\mathfrak{m}$ is a reductive Lie subgroup of $\mathfrak{g}$ and $\mathfrak{p}=\mathbb{C}^{2} \otimes V_{M}$ where $V_{M}$ is a self dual representation of $\mathfrak{m}$. $\mathfrak{k}$ contains a Cartan subalgebra $\mathfrak{h}$ of $\mathfrak{g}$. We choose a positive root system $\Phi^{+}$with respect to $\mathfrak{h}$ such that the $\mathfrak{s u}_{2}$ in $\mathfrak{k}$ corresponds to the highest root $\widetilde{\alpha}$. Denote this Lie algebra by $\mathfrak{s u}_{2}(\widetilde{\alpha}) . \mathfrak{t}_{1}=\mathfrak{h} \cap \mathfrak{s u}_{2}(\widetilde{\alpha})$ is a Cartan subalgebra of $\mathfrak{s u}_{2}(\widetilde{\alpha})$. Then $\mathfrak{l}=\mathfrak{t}_{1} \oplus \mathfrak{m}$ is a Levi subalgebra of a maximal parabolic subalgebra $\mathfrak{q}$ whose nilpotent radical $\mathfrak{u}$ is a Heisenberg Lie algebra. The one dimensional center of $\mathfrak{u}$ is spanned by the highest root space $\mathfrak{g}_{\widetilde{\alpha}}$ and let $\mathfrak{u} /[\mathfrak{u}, \mathfrak{u}]=V_{M}$ denote the representation of $\mathfrak{m} . \overline{\mathfrak{q}}=\mathfrak{l} \oplus \overline{\mathfrak{u}}$ will denote the opposite parabolic subalgebra.

Let $G(\mathbb{C})$ be a complex simply connected simple Lie group with Lie algebra $\mathfrak{g}$ and let $G_{0}$ be a real form of $G(\mathbb{C})$ having Lie algebra $\mathfrak{g}_{0}$. We denote the real Lie subgroups in $G(\mathbb{C})$ corresponding to the various real forms of the Lie subalgebras $\mathfrak{m}_{0}, \mathfrak{l}_{0}$ and $\mathfrak{k}_{0}$ by $M, L$ and $K_{0}=S U_{2}(\widetilde{\alpha}) \times_{\mu_{2}} M$ respectively. Here $K_{0}$ is the maximal compact subgroup of $G_{0}$ and $S U_{2}(\widetilde{\alpha})=M_{1}$ in $\S 1.1$. Let $G$ denote the double cover of $G_{0}$ with maximal compact subgroup $K=S U_{2}(\widetilde{\alpha}) \times M$. We will call $G \times H$ a quaternionic Lie group if $G$ is a quaternionic simple Lie group and $H$ is a compact Lie group.

Set $2 d=\operatorname{dim} V_{M}$. If $\mathfrak{g}$ is of type $D_{4}, F_{4}, E_{6}, E_{7}, E_{8}$, then $d=3 s+4$ where $s=0,1,2,4,8$ respectively. We tabulate some of the $M(\mathbb{C})$ and $V_{M}$ in Table 1 below.

\begin{tabular}{l|l|l|l} 
& $G_{0}$ & $M(\mathbb{C})$ & $V_{M}$ \\
\hline & $\operatorname{Spin}(d, 4), d \geq 4$ & $S L_{2} \times \operatorname{Spin}(d)$ & $\mathbb{C}^{2} \otimes \mathbb{C}^{d}$ \\
$\left(\mathrm{e}_{1}\right)$ & $F_{4,4}$ & $S p_{6}$ & $\pi\left(\varpi_{3}\right)$ \\
$\left(\mathrm{e}_{2}\right)$ & $E_{6,4} \rtimes \mathbb{Z} / 2 \mathbb{Z}$ & $S L_{6} \rtimes \mathbb{Z} / 2 \mathbb{Z}$ & $\pi\left(\varpi_{3}\right)$ \\
$\left(\mathrm{e}_{4}\right)$ & $E_{7,4}$ & $\operatorname{Spin}(12)$ & $\pi\left(\varpi_{6}\right)$ \\
$\left(\mathrm{e}_{8}\right)$ & $E_{8,4}$ & simply connected $E_{7}$ & $\pi\left(\varpi_{1}\right)$
\end{tabular}

Table 1.

2.3. We will define and review the properties of quaternionic representations. We refer to $[\mathbf{S 1}],[\mathbf{W} \mathbf{1}],[\mathbf{W} \mathbf{2}],[\mathbf{G W} \mathbf{1}],[\mathbf{G W 2}, \S 5]$ and Theorem 3.3.1 of $[\mathbf{L 2}]$ for proofs and details.

Let $W[k]=e^{-k \widetilde{\alpha} / 2} \otimes W$ be an irreducible finite dimensional representation of $L=U_{1} \times M$. We extend $W[k]$ trivially to a representation of $\overline{\mathfrak{q}}$ and denote

$$
\mathbf{H}(G, W[k])=\mathbf{H}(W[k]):=\Gamma_{K / L}^{1}\left(\operatorname{Hom}_{\mathcal{U}(\overline{\mathfrak{q}})}(\mathcal{U}(\mathfrak{g}), W[k])_{L}\right)
$$

as the Harish-Chandra module of $G$ where $\Gamma^{1}$ is the first Zuckerman derived functor. It has infinitesimal character $\mu+\rho(G)-k \frac{\widetilde{\alpha}}{2}$. If $k \geq 2$, then $\mathbf{H}(W[k])$ 
has $K$-types $\left(K=S U_{2}(\widetilde{\alpha}) \times M\right)$

$$
\sum_{n=0}^{\infty} S_{\widetilde{\alpha}}^{k-2+n}\left(\mathbb{C}^{2}\right) \otimes\left(\operatorname{Sym}^{n}\left(V_{M}\right) \otimes W\right) .
$$

It contains a unique irreducible $(\mathfrak{g}, K)$-submodule denoted by $\sigma(G, W[k])$ which is generated by the translates of the lowest $K$-types

$$
S_{\widetilde{\alpha}}^{k-2}\left(\mathbb{C}^{2}\right) \otimes W .
$$

We will call $\mathbf{H}(G, W[k])$ and $\sigma(G, W[k])$ quaternionic representations.

\section{Restrictions.}

3.1. Let $G_{0}$ be one of the four exceptional groups given in Table $1\left(\mathrm{e}_{s}\right)$ indexed by $s=1,2,4,8$. Let $G$ be its double cover.

3.2. Suppose $G^{\prime}$ is a quaternionic real Lie subgroup of $G$ containing $S U_{2}(\widetilde{\alpha})$. We have correspondingly $K^{\prime}=G^{\prime} \cap K, M^{\prime}=G^{\prime} \cap M$ and the Lie algebras $\mathfrak{g}^{\prime}, \mathfrak{m}^{\prime}, \mathfrak{u}^{\prime}=\mathfrak{u} \cap \mathfrak{g}^{\prime}$. Write $\mathfrak{u}=\mathfrak{u}^{\prime}+V_{0}$. We have $V_{M^{\prime}} \subset V_{M}$ and we define $V_{0}=V_{M} / V_{M^{\prime}}$ as a representation of $M^{\prime}$.

We will use $\operatorname{Res}_{G^{\prime}}^{G} \sigma$ to denote the restriction of a Harish-Chandra module $\sigma$ of $G$ to that of $G^{\prime}$.

3.3. First we review the work of $[\mathbf{G W 2}]$. Denote $\mathbf{H}_{k}:=\mathbf{H}(G, \mathbb{C}[k])$ and its unique submodule $\sigma(G, \mathbb{C}[k])$ by $\sigma_{k} . \mathbf{H}_{k}$ is irreducible and unitarizable if $k \geq 3 s+4$. If $k>6 s+8$, then it belongs to the discrete series. We recall Corollary 4.2.2 of [L2].

Proposition 3.3.1. There exists a filtration $H_{n}$ of $\mathbf{H}_{k}$ such that $H_{n+1} / H_{n}$ $=\mathbf{H}\left(G^{\prime}, S^{n}\left(V_{0}\right)[n+k]\right)$. If $\mathbf{H}_{k}$ is unitarizable, then

$$
\operatorname{Res}_{G^{\prime}}^{G} \mathbf{H}_{k}=\sum_{n=0}^{\infty} \mathbf{H}\left(G^{\prime}, S^{n}\left(V_{0}\right)[n+k]\right)
$$

and each summand on the right is irreducible and unitarizable.

3.4. Since $V_{M}$ is a self dual representation of $M$, we identify $V_{M}$ with its dual. In $\S 1.2$ we remarked that $\mathbb{P} V_{M}$ is a union of four $M(\mathbb{C})$-orbits. One of the orbit is Zariski dense and we denote the Zariski closure of the other three non-dense orbits by $X, Y$ and $Z$ satisfying (1). We will describe these three orbits in greater detail in $\S 5$.

Let $(k, m, \mathcal{O})$ be one of the three following sets of data:

$$
(3 s+3,4, X), \quad(2 s+2,3, Y), \quad(s+2,2, Z) .
$$

Let $I^{\bullet}(\mathcal{O})=\bigoplus_{n>m} I^{n}(\mathcal{O}),\left(I^{m} \neq 0\right)$ be the homogeneous ideal of $\mathcal{O}$ in $\mathbb{P} V_{M}$ and $A^{\bullet}(\mathcal{O})=\bigoplus A^{n}(\mathcal{O})$ be its coordinate ring. Then $I^{\bullet}$ is generated as a $S^{\bullet}\left(V_{m}\right)$ module by $I^{m}(\mathcal{O})$ and each graded piece $A^{n}(\mathcal{O})$ is a representation of $M$. 
Gross and Wallach showed that $\sigma_{k}$ is a unitarizable proper submodule of $\mathbf{H}_{k}$ with $K$ types given in (2). Furthermore it satisfies an exact sequence

$$
0 \rightarrow \sigma_{k} \rightarrow \mathbf{H}(G, \mathbb{C}[k]) \stackrel{\phi}{\rightarrow} \mathbf{H}\left(G, I^{m}(\mathcal{O})[k+m]\right)
$$

As a representation of $M(\mathbb{C}), I^{m}(\mathcal{O})=\mathbb{C}, V_{M}$ and $\mathfrak{m}$ respectively for the three values of $k$. When $k=3 s+3, \phi$ in (11) is a surjection (cf. $\S 8$ of [GW2]). We will follow the notation of $[\mathbf{G W} \mathbf{1}]$ and denote the three representations $\sigma_{3 s+3}, \sigma_{2 s+2}$ and $\sigma_{s+2}$ by $\sigma_{X}, \sigma_{Y}$ and $\sigma_{Z}$ respectively.

The annihilator ideal of $\sigma_{Z}$ is the Joseph ideal in $\mathcal{U}(\mathfrak{g})$ so $\sigma_{Z}$ is called the minimal representation of $G$. It has $K$-types

$$
\sum_{n=0}^{\infty} S_{\widetilde{\alpha}}^{s+n}\left(\mathbb{C}^{2}\right) \otimes \pi_{M}(n \lambda)
$$

where $\lambda$ is the highest weight of $V_{M}$. Note that all the representations descend to representations of $G_{0}$ except $\sigma_{X}$ for groups of type $E_{n}$ and $\sigma_{Z}$ of $\widetilde{F}_{4,4}$.

3.5. The inclusion $I^{m}(\mathcal{O}) \subset S^{m}\left(V_{M}\right)$ gives rise to the following natural maps of $M$-modules:

$$
\operatorname{Sym}^{n-m}\left(V_{M}\right) \otimes I^{m}(\mathcal{O}) \rightarrow \operatorname{Sym}^{n-m}\left(V_{M}\right) \otimes \operatorname{Sym}^{m}\left(V_{M}\right) \rightarrow \operatorname{Sym}^{n}\left(V_{M}\right) .
$$

Let $r_{n}^{\prime}$ denote the composite of the above maps. The direct sum $V_{M}=\mathfrak{u}^{\prime} \oplus V_{0}$ (cf. §3.2) induces a natural map of $M^{\prime}$-modules

$$
r_{n}^{\prime \prime}: \operatorname{Sym}^{n}\left(V_{M}\right) \rightarrow \operatorname{Sym}^{n}\left(V_{0}\right) .
$$

We define $r_{n}=r_{n}^{\prime \prime} \circ r_{n}^{\prime}$ for $n \geq m$. For $0 \leq n<m$, we set $r_{n}$ to be the zero map into $\operatorname{Sym}^{n}\left(V_{0}\right)$. Let $R_{n}$ denote the cokernel of $r_{n}$ and let $R_{\bullet}:=\bigoplus_{n=0}^{\infty} R_{n}$. Note that $R_{n}$ is a representation of $M^{\prime}$ and we write

$$
R_{n}=\sum_{j} W_{n, j}
$$

where $W_{n, j}$ are the irreducible subrepresentations of $M^{\prime}$.

Let $\mathcal{O}^{\prime}=\mathcal{O} \cap \mathbb{P} V_{0}$ and we denote its coordinate ring in $\mathbb{P} V_{0}$ by $A^{\bullet}\left(\mathcal{O}^{\prime}\right)=$ $\bigoplus A^{n}\left(\mathcal{O}^{\prime}\right)$. Then $\mathcal{O}^{\prime}$ is cut out by $r_{m}\left(I^{m}(\mathcal{O})\right)$ and $R_{\bullet} / \operatorname{Nil}\left(R_{\bullet}\right)=A^{\bullet}\left(\mathcal{O}^{\prime}\right)$.

We can now state Theorem 3.3.1 and Corollary 2.8.1 of [L3]. 


\section{Theorem 3.5.1.}

(a)

$$
\operatorname{Res}_{G^{\prime}}^{G} \sigma_{k}=\sum_{n=0}^{\infty} \sigma\left(G^{\prime}, R_{n}[k+n]\right)=\sum_{n=0}^{\infty} \sum_{j} \sigma\left(G^{\prime}, W_{n, j}[k+n]\right) .
$$

(b)

$$
\operatorname{Res}_{G^{\prime}}^{G} \sigma_{k} \supseteq \sum_{n=0}^{\infty} \sigma\left(G^{\prime}, A^{n}\left(\mathcal{O}^{\prime}\right)[k+n]\right) .
$$

Equality holds if and only if $r_{m}\left(I^{m}(\mathcal{O})\right)$ generates the ideal of $\mathcal{O}^{\prime}$.

(c) If $r_{m}$ is surjective, then $r_{n}$ is surjective for $n \geq m$ and

$$
\operatorname{Res}_{G^{\prime}}^{G} \sigma_{k}=\sum_{n=0}^{m-1} \sigma\left(G^{\prime}, S^{n} V_{0}[k+n]\right)
$$

\section{The main results.}

4.1. In this section we will state the main results on the restrictions of the quaternionic representations $\sigma_{\mathcal{O}}$ of the exceptional group $G$ to its subgroup $G^{\prime}$. The proofs will be given in the later sections. We will replace $G$ by $G_{0}$ if the representation descends to $G_{0}$. If $\mathbf{H}\left(G^{\prime}, W[n]\right)$ appears in the restriction formula, it means that $\mathbf{H}\left(G^{\prime}, W[n]\right)$ is irreducible and its $K$-types are given by $(9)$.

4.2. Let $G=\widetilde{F}_{4,4} \supset G^{\prime}=\widetilde{\operatorname{Spin}}(5,4)$ and $M^{\prime}=S U_{2} \times \operatorname{Spin}(5)$.

Let $V_{m, n}=S^{m}\left(\mathbb{C}^{2}\right) \otimes \pi_{\text {Spin (5) }}\left(n \varpi_{2}\right)$ be the representation of $M^{\prime}$.

\section{Theorem 4.2.1.}

(a) $\quad \operatorname{Res}_{G^{\prime}}^{G} \sigma_{Z}=\sigma\left(G^{\prime}, \mathbb{C}[3]\right)+\sigma\left(G^{\prime}, V_{0,1}[4]\right)$.

(b) $\quad \operatorname{Res}_{\operatorname{Spin}(5,4)}^{F_{4,4}} \sigma_{Y}=\sum_{n=0}^{\infty} \sigma\left(\operatorname{Spin}(5,4), V_{0, n}[4+n]\right)$.

(c) $\quad \operatorname{Res}_{\operatorname{Spin}(5,4)}^{F_{4,4}} \sigma_{X}=\mathbf{H}(\operatorname{Spin}(5,4), \mathbb{C}[6])+\sum_{n=1}^{\infty} \sigma\left(\operatorname{Spin}(5,4), V_{0, n}[6+n]\right)$.

(d) $\operatorname{Res}_{G^{\prime}}^{G} \mathbf{H}(G, \mathbb{C}[k])=\sum_{n=0}^{\infty} \mathbf{H}\left(G^{\prime}, V_{0, n}[k+n]\right)$ if $k \geq 7$.

If $n \geq 1$ then the summands in (c) satisfy the following exact sequence:

$$
0 \rightarrow \sigma\left(F_{4,4}, V_{0, n}[6+n]\right) \rightarrow \mathbf{H}\left(F_{4,4}, V_{0, n}[6+n]\right) \rightarrow \mathbf{H}\left(F_{4,4}, V_{0, n}[9+n]\right) \rightarrow 0
$$


4.3. Suppose $G=\widetilde{F}_{4,4}$ and $G^{\prime}=\widetilde{\operatorname{Spin}}(4,4)$. The maximal compact subgroup of $G^{\prime}$ is $S U_{2}(\widetilde{\alpha}) \times M^{\prime}$ where $M^{\prime}=S U_{2}(A) \times S U_{2}(B) \times S U_{2}(C)$. Here $\widetilde{\alpha}, A, B, C$ are the (orthogonal) compact roots of $\widetilde{\operatorname{Spin}}(4,4)$. Let

$$
S(a, b, c):=S_{A}^{a}\left(\mathbb{C}^{2}\right) \otimes S_{B}^{b}\left(\mathbb{C}^{2}\right) \otimes S_{C}^{c}\left(\mathbb{C}^{2}\right)
$$

denote the irreducible representation of $M^{\prime}$.

\section{Theorem 4.3.1.}

(a) $\begin{aligned} \operatorname{Res}_{G^{\prime}}^{G} \sigma_{Z}= & \sigma\left(G^{\prime}, \mathbb{C}[3]\right)+\sigma\left(G^{\prime}, S(1,0,0)[4]\right) \\ & +\sigma\left(G^{\prime}, S(0,1,0)[4]\right)+\sigma\left(G^{\prime}, S(0,0,1)[4]\right) .\end{aligned}$

(b) $\operatorname{Res}_{\operatorname{Spin}(4,4)}^{F_{4,4}} \sigma_{Y}=\sum_{a b c=0} \sigma(\operatorname{Spin}(4,4), S(a, b, c)[4+a+b+c])$.

(c) $\quad \operatorname{Res}_{\operatorname{Spin}(4,4)}^{F_{4,4}} \sigma_{X}=\sum_{a b c=0} \mathbf{H}(\operatorname{Spin}(4,4), S(a, b, c)[6+a+b+c])$

$$
+\sum_{a, b, c>0} \sigma(\operatorname{Spin}(4,4), S(a, b, c)[6+a+b+c]) .
$$

(d) $\quad \operatorname{Res}_{G^{\prime}}^{G} \mathbf{H}(G, \mathbb{C}[k])=\sum_{a, b, c \geq 0} \mathbf{H}\left(G^{\prime}, S(a, b, c)[k+a+b+c]\right)$ if $k \geq 7$.

When $a, b, c$ are strictly positive, the summands in (c) satisfy the following exact sequence:

$$
\begin{aligned}
0 \rightarrow \sigma(\operatorname{Spin} & (4,4), S(a, b, c)[6+a+b+c]) \\
& \rightarrow \mathbf{H}(\operatorname{Spin}(4,4), S(a, b, c)[6+a+b+c]) \\
& \rightarrow \mathbf{H}(\operatorname{Spin}(4,4), S(a-1, b-1, c-1)[7+a+b+c]) \rightarrow 0
\end{aligned}
$$

4.4. Note that $\widetilde{\operatorname{Spin}}(4,4)$ and $\widetilde{\operatorname{Spin}}(5,4)$ are components of dual pairs in $(3)$ and (4). In $\S 6$ we will describe the action of $(\mathbb{Z} / 2 \mathbb{Z})^{2}$ and $(\mathbb{Z} / 2 \mathbb{Z})^{3}$ on the summands. In particular Theorems 4.2.1(a) and 4.3.1(a) give the dual pairs correspondences of the minimal representation $\sigma_{Z}$ of $\widetilde{F}_{4,4}$. The $K$-types of summands are given in Theorem 6.8.1 and 6.9.1.

4.5. Let $G=\widetilde{E}_{6,4} \rtimes \mathbb{Z} / 2 \mathbb{Z}$ and $G^{\prime}=\widetilde{F}_{4,4} \times \mathbb{Z} / 2 \mathbb{Z}$. Let $\chi$ be the nontrivial character of $\mathbb{Z} / 2 \mathbb{Z}$. 


\section{Theorem 4.5.1.}

(a) $\quad \operatorname{Res}_{F_{4,4} \times \mathbb{Z} / 2 \mathbb{Z}}^{E_{6,4} \times \mathbb{Z} / 2 \mathbb{Z}} \sigma_{Z}=\sigma\left(F_{4,4}, \mathbb{C}[4]\right) \otimes \chi^{0}+\sigma\left(F_{4,4}, \mathbb{C}^{6}[5]\right) \otimes \chi$.

(b)

$$
\begin{aligned}
\operatorname{Res}_{F_{4,4} \times \mathbb{Z} / 2 \mathbb{Z}}^{E_{6,4} \times \mathbb{Z} / 2 \mathbb{Z}} \sigma_{Y}= & \sum_{n=0}^{\infty} \sigma\left(F_{4,4}, S^{n}\left(\mathbb{C}^{6}\right)[6+n]\right) \otimes \chi^{n} . \\
\operatorname{Res}_{G^{\prime}}^{G} \sigma_{X}= & \mathbf{H}\left(\widetilde{F}_{4,4}, \mathbb{C}[9]\right) \otimes \chi^{0}+\mathbf{H}\left(\widetilde{F}_{4,4}, \mathbb{C}^{6}[10]\right) \otimes \chi \\
& +\sum_{n=2}^{\infty} \sigma\left(\widetilde{F}_{4,4}, S^{n}\left(\mathbb{C}^{6}\right)[9+n]\right) \otimes \chi^{n} .
\end{aligned}
$$

(d) $\operatorname{Res}_{G^{\prime}}^{G} \mathbf{H}(G, \mathbb{C}[k])=\sum_{n=0}^{\infty} \mathbf{H}\left(\widetilde{F}_{4,4}, S^{n}\left(\mathbb{C}^{6}\right)[10+n]\right) \otimes \chi^{n}$ if $k \geq 10$.

If $n \geq 2$ then the summands in (c) satisfy the following exact sequence:

$$
\begin{aligned}
0 \rightarrow \sigma\left(\widetilde{F}_{4,4}, S^{n}\left(\mathbb{C}^{6}\right)[9+n]\right) \rightarrow \mathbf{H}\left(\widetilde{F}_{4,4}, S^{n}\left(\mathbb{C}^{6}\right)[9+n]\right) & \\
& \rightarrow \mathbf{H}\left(\widetilde{F}_{4,4}, S^{n-2}\left(\mathbb{C}^{6}\right)[11+n]\right) \rightarrow 0
\end{aligned}
$$

4.6. Each of the summands of $\sigma_{X}$ in Theorems 4.2.1(c), 4.3.1(c) and 4.5.1(c) satisfies a short exact sequence. The $K^{\prime}$-types of the middle and the last term of the exact sequence is given by (9). Hence it is possible to determine the $K^{\prime}$-types, the Gelfand-Kirillov dimensions and the Bernstein degrees of the summands.

4.7. Let $G=\widetilde{E}_{7,4}, G^{\prime}=\widetilde{E}_{6,4} \times_{\mu_{3}} U_{1}$ and $M^{\prime}=S U_{6} \times_{\mu_{3}} U_{1}$.

Let $V_{a, b}=\pi_{S U_{6}}\left(a \varpi_{1}+b \varpi_{6}\right)$.

\section{Theorem 4.7.1.}

(a) $\operatorname{Res}_{E_{6,4} \times U_{1}}^{E_{7,4}} \sigma_{Z}=\sum_{a, b \geq 0, a b=0} \sigma\left(E_{6,4}, V_{a, b}[6+a+b]\right) \otimes \chi_{1}^{a-b}$.

(b) $\operatorname{Res}_{E_{6,4} \times U_{1}}^{E_{7,4}} \sigma_{Y}=\mathbf{H}\left(E_{6,4}, \mathbb{C}[12]\right) \otimes \chi_{1}^{0}$

$$
+\sum_{a, b \geq 0} \sigma\left(E_{6,4}, V_{a, b}[10+a+b]\right) \otimes \chi_{1}^{a-b} .
$$

(c) $\operatorname{Res}_{G^{\prime}}^{G} \sigma_{X}=\sum_{n=0,1} \sum_{a, b \geq 0} \mathbf{H}\left(\widetilde{E}_{6,4}, V_{a, b}[15+2 n+a+b]\right) \otimes \chi_{1}^{a-b}$.

(d) $\operatorname{Res}_{G^{\prime}}^{G} \mathbf{H}(G, \mathbb{C}[k])=\sum_{a, b, n \geq 0} \mathbf{H}\left(\widetilde{E}_{6,4}, V_{a, b}[k+2 n+a+b]\right) \otimes \chi_{1}^{a-b}$

$$
\text { if } k \geq 16 \text {. }
$$

4.8. Let $G=\widetilde{E}_{8,4}, G^{\prime}=\widetilde{E}_{7,4} \times_{\mu_{2}} S U_{2}$ and $M^{\prime}=\operatorname{Spin}(12) \times_{\mu_{2}} S U_{2}$. Let $V_{a, b}=\pi_{\text {Spin (12) }}\left(a \varpi_{1}+b \varpi_{2}\right)$. 


\section{Theorem 4.8.1.}

(a) $\operatorname{Res}_{E_{7,4} \times S U_{2}}^{E_{8,4}} \sigma_{Z}=\sum_{n=0}^{\infty} \sigma\left(E_{7,4}, V_{n, 0}[10+n]\right) \otimes S^{n}\left(\mathbb{C}^{2}\right)$.

(b) $\operatorname{Res}_{E_{7,4} \times S U_{2}}^{E_{8,4}} \sigma_{Y}=\sum_{a+2 b+2 c=n, b c=0} \sigma\left(E_{7,4}, V_{a, c}[18+n]\right) \otimes S^{a+2 b}\left(\mathbb{C}^{2}\right)$.

(c) $\quad \operatorname{Res}_{G^{\prime}}^{G} \sigma_{X}=\sum{ }^{*} \mathbf{H}\left(\widetilde{E}_{7,4}, V_{a+2 d, c}[27+n]\right) \otimes S^{a+2 b}\left(\mathbb{C}^{2}\right)$.

(d) $\operatorname{Res}_{G^{\prime}}^{G} \mathbf{H}(G, \mathbb{C}[k])=\sum_{m=0}^{\infty} \sum{ }^{*} \mathbf{H}\left(\widetilde{E}_{7,4}, V_{a+2 d, c}[k+n+4 m]\right) \otimes$

$$
\otimes S^{a+2 b}\left(\mathbb{C}^{2}\right) \text { if } k \geq 28
$$

where the summation $\sum^{*}$ is taken over all nonnegative integers $a, b, c, d, n$ satisfying the relations

$$
n-2 a \leq a+2 b+2 c+4 d \leq n, \quad c d=0, \quad a \equiv n \quad \bmod (2) .
$$

4.9. Some of the restrictions stated in this section are known. We have included them for the sake of completeness. Moreover they follow with little additional effort from the proofs of the rest of the statements. The following results are known:

(i) Theorems 4.5.1(a) and 4.7.1(a) are unpublished results of B. Gross [G]. See $\S 6$ [GW1] for Theorem 4.8.1(a). The method of proof is by considering the decompositions of the $K$-types. Also see Theorem 12.1 .1 [L2].

(ii) See $\S 4.6$ of [L2] for Theorems 4.3.1(c) and (d).

4.10. Using the above theorems, we will deduce the following dual pair correspondence in $\S 9.4$ :

Corollary 4.10.1.

$$
\operatorname{Res}_{E_{6,4} \times \mu_{3} S U_{3}}^{E_{8,4}} \sigma_{Z}=\sum_{a, b \geq 0} \Theta(a, b) \otimes \pi_{S U_{3}}\left(a \varpi_{1}+b \varpi_{2}\right)
$$

where

$$
\Theta(a, b)= \begin{cases}\sigma\left(E_{6,4}, \pi_{S U_{6}}\left(a \varpi_{1}+b \varpi_{5}\right)[a+b+10]\right) & \text { if }(a, b) \neq(0,0) \\ \mathbf{H}\left(E_{6,4}, \mathbb{C}[10]\right) \oplus \mathbf{H}\left(E_{6,4}, \mathbb{C}[12]\right) & \text { if }(a, b)=(0,0) .\end{cases}
$$

The dual pair correspondence of $\operatorname{Spin}(4,4) \times{ }_{\mu_{2}^{2}} U_{1}^{2} \in E_{6,4}$ will be given in Theorem 7.3.1. 
4.11. The proofs of part (c) of Theorems 4.2.1 to 4.8.1 are similar. By (11) we have an exact sequence

$$
0 \rightarrow \sigma_{X} \rightarrow \mathbf{H}(G, \mathbb{C}[d-1]) \rightarrow \mathbf{H}(G, \mathbb{C}[d+3]) \rightarrow 0 .
$$

The term on the right is irreducible and unitarizable. By Proposition 3.3.1 the restriction of the last term to $G^{\prime}$ decomposes into

$$
\sum_{n=0}^{\infty} \mathbf{H}\left(G^{\prime}, S^{n} V_{0}[d+3+n]\right) \text {. }
$$

Applying the filtration in Proposition 3.3.1 to the middle and the last term of (18) gives a homomorphism of graded modules

$$
0 \rightarrow \operatorname{Res}_{G^{\prime}}^{G} \sigma_{X} \rightarrow \sum_{n=0}^{\infty} \mathbf{H}\left(G^{\prime}, S^{n} V_{0}[d-1+n]\right) \rightarrow \sum_{n=0}^{\infty} \mathbf{H}\left(G^{\prime}, S^{n} V_{0}[d+3+n]\right) \rightarrow 0
$$

One can show that the above sequence is an exact sequence. We shall use (20) to prove part (c) of the above theorems.

\section{Orbits computations.}

5.1. In this section we will give a realization of the $M(\mathbb{C})$ action on $\mathbb{P} V_{M}$ and describe its subvarieties $X, Y$ and $Z$ (cf. $§ 1.2$ ). Please refer to [Ba], [Kim], [J1], [GW2] and [GL] for more details.

5.2. Let $s=1,2,4$, or 8 and let $\mathcal{K}=\mathcal{K}_{s}$ denote the composition algebra over $\mathbb{C}$ of dimension $s$. Then up to isomorphism, $\mathcal{K}_{1}=\mathbb{C}, \mathcal{K}_{4}$ is the set of 2 by 2 complex matrices $M_{2}(\mathbb{C}), \mathcal{K}_{2}$ is the subset of diagonal elements in $M_{2}(\mathbb{C})$ and $\mathcal{K}_{8}$ is the set of Cayley numbers [J2]. Each algebra has an anti-automorphism $z \mapsto \bar{z}$ called conjugation such that $N(z):=z \bar{z}=\bar{z} z$ is a nondegenerate bilinear form on $\mathcal{K}$. Moreover $N\left(z z^{\prime}\right)=N(z) N\left(z^{\prime}\right)$. Define $\operatorname{tr}(z)=z+\bar{z}$ and $\left\langle z, z^{\prime}\right\rangle=\operatorname{tr}\left(z \overline{z^{\prime}}\right)$. There are obvious embeddings $\mathcal{K}_{1} \subset \mathcal{K}_{2} \subset \mathcal{K}_{4}$

5.3. Let $\left(U_{0},\langle\rangle,\right)$ be the 3 dimensional complex inner product space with orthonormal basis $\left\{e_{1}, e_{2}, e_{3}\right\}$. Then $\mathcal{K}_{8}$ can be realized as elements of the form

$$
x=\left(a, d ; v_{1}, v_{2}\right):=\left(\begin{array}{cc}
a & v_{1} \\
v_{2} & d
\end{array}\right)
$$

where $a, d \in \mathbb{C}$ and $v_{1}, v_{2} \in U_{0}$. The multiplication is given in [J2, p. 142] and $N(x)=a d-\left\langle v_{1}, v_{2}\right\rangle$. We define an action of $g \in S L\left(U_{0}\right)$ on $\mathcal{K}_{8}$ by

$$
g:\left(a, d ; v_{1}, v_{2}\right) \mapsto\left(a, d ; h v_{1},{ }^{t} h^{-1} v_{2}\right)
$$

We embed $\mathcal{K}_{4} \subset \mathcal{K}_{8}$ by $\left(\begin{array}{ll}a & b \\ c & d\end{array}\right) \mapsto\left(\begin{array}{cc}a & b e_{1} \\ c e_{1} & d\end{array}\right)$. 
5.4. Let $\mathcal{J}=\mathcal{J}(\mathcal{K})$ be the Jordan algebra consisting of 3 by 3 Hermitian symmetric matrices of the form

$$
J=\left(\gamma_{1}, \gamma_{2}, \gamma_{3} ; c_{1}, c_{2}, c_{3}\right):=\left(\begin{array}{ccc}
\gamma_{1} & c_{3} & \overline{c_{2}} \\
\overline{c_{3}} & \gamma_{2} & c_{1} \\
c_{2} & \overline{c_{1}} & \gamma_{3}
\end{array}\right)
$$

where $\gamma_{i} \in \mathbb{C}$ and $c_{i} \in \mathcal{K}$. The composition in $\mathcal{J}$ is given by $J_{1} \circ J_{2}=$ $\frac{1}{2}\left(J_{1} J_{2}+J_{1} J_{2}\right)$. Define an inner product on $\mathcal{J}$ given by $\langle X, Y\rangle=\operatorname{Tr}(X \circ Y)$ where $\operatorname{Tr}$ denotes the usual trace of matrices. There is a cubic form

$$
\operatorname{det}(J)=\gamma_{1} \gamma_{2} \gamma_{3}-\gamma_{1} N\left(c_{1}\right)-\gamma_{2} N\left(c_{2}\right)-\gamma_{3} N\left(c_{3}\right)+\operatorname{tr}\left(c_{1}\left(c_{2} c_{3}\right)\right)
$$

on $\mathcal{J}$ which induces a trilinear form on $\mathcal{J}$ such that $(J, J, J)=\operatorname{det} J$. For $\mathcal{J}\left(\mathcal{K}_{1}\right)$ and $\mathcal{J}\left(\mathcal{K}_{2}\right)$, det is the usual determinant of 3 by 3 matrices. Finally we define a bilinear map $\mathcal{J} \times \mathcal{J} \longrightarrow \mathcal{J},\left(J, J^{\prime}\right) \mapsto J \times J^{\prime}$ such that in the notation of $(23)$

$$
\begin{aligned}
J \times J= & \left(\gamma_{2} \gamma_{3}-N\left(c_{1}\right), \gamma_{3} \gamma_{1}-N\left(c_{2}\right), \gamma_{1} \gamma_{2}-N\left(c_{3}\right) ;\right. \\
& \left.\overline{c_{2} c_{3}}-\gamma_{1} c_{1}, \overline{c_{3} c_{1}}-\gamma_{2} c_{2}, \overline{c_{1} c_{2}}-\gamma_{3} c_{3}\right) .
\end{aligned}
$$

$J \neq 0$ is said to have $\operatorname{rank} 1$ if $J \times J=0 . J$ has rank 0 if $J=0 . J \neq 0$ has rank 2 if $\operatorname{det}(J)=0$ and it is not of rank 1.

Define

$$
V_{M}:=\mathbb{C} \oplus \mathcal{J} \oplus \mathcal{J} \oplus \mathbb{C}
$$

and we denote a vector in $V_{M}$ by $\left(\xi, J, J^{\prime}, \xi^{\prime}\right)$. There is a realization of the $M(\mathbb{C})$ action on $V_{M}$ (see $[\mathrm{Ba}]$ ). Let $p: V_{M} \backslash\{0\} \rightarrow \mathbb{P} V_{M}$ be the canonical projection.

We refer to (23) and define

$$
\mathcal{J}_{1}:=\left\{\left(0,0,0 ; c_{1}, 0,0\right) \in \mathcal{J}: c_{1} \in \mathcal{K}\right\} .
$$

Similarly we define $\mathcal{J}_{2}$ and $\mathcal{J}_{3}$. Define $W_{i}=\left\{\left(0, J, J^{\prime}, 0\right) \in V_{M}: J, J^{\prime} \in \mathcal{J}_{i}\right\}$ for $i=1,2,3$. We will need these definitions in $\S 6$ and $\S 7$.

5.5. The smallest orbit $Z$ is generated by the highest weight vector spanned by $p(1,0,0,0)$. The stabilizer of $p(1,0,0,0)$ is a maximal abelian parabolic $Q=L^{\prime} \rtimes N^{\prime}$ in $M(\mathbb{C})$. We denote $\bar{Q}^{\prime}=L \rtimes \bar{N}^{\prime}$ to be the opposite parabolic subgroup. Then $\bar{Q}^{\prime}$ stabilizes the flag

$$
\mathbb{C} \subset \mathcal{J} \oplus \mathbb{C} \subset \mathcal{J} \oplus \mathcal{J} \oplus \mathbb{C} \subset V_{M} .
$$

There is a bijection $\mathcal{J}(\mathcal{K}) \rightarrow \bar{N}^{\prime}$ given by $B \mapsto p_{B}$ where $p_{B}$ acts on $V_{M}$ by (see $[\mathrm{Kim}]$ )

$$
p_{B}:\left(0, J, J^{\prime}, 0\right) \mapsto\left(0, J, J^{\prime}+2 B \times J,(B, B, J)+\left(B, J^{\prime}\right)\right) .
$$

We recall a version of Lemma 7.5 of [MS]. 
Lemma 5.5.1. Representatives of the $\bar{N}^{\prime}$-orbits on $Z$ are

$$
\begin{array}{cc}
v_{1}=p(1,0,0,0) & v_{2}=p(0, J, 0,0) \\
v_{3}=p\left(0,0, J^{\prime}, 0\right) & v_{4}=p(0,0,0,1)
\end{array}
$$

where $J$ and $J^{\prime}$ are rank 1 elements in $\mathcal{J}$.

5.6. The variety $X$ is the hypersurface given by the degree 4 polynomial (cf. $[\mathbf{J} 1])$

$f_{4}\left(\xi, J, J^{\prime}, \xi^{\prime}\right)=\left\langle J \times J, J^{\prime} \times J^{\prime}\right\rangle-\xi \operatorname{det}(J)-\xi^{\prime} \operatorname{det}\left(J^{\prime}\right)-\frac{1}{4}\left(\left\langle J, J^{\prime}\right\rangle-\xi \xi^{\prime}\right)^{2}$.

If $J_{1}=(0,0,0 ; x, y, z)$ and $J_{2}=\left(0,0,0 ; x^{\prime}, y^{\prime}, z^{\prime}\right)$, then

$$
\begin{aligned}
f_{4}\left(0, J_{1}, J_{2}, 0\right)= & N(x) N\left(x^{\prime}\right)+N(y) N\left(y^{\prime}\right)+N(z) N\left(z^{\prime}\right)+\left\langle y z, y^{\prime} z^{\prime}\right\rangle \\
& +\left\langle z x, z^{\prime} x^{\prime}\right\rangle+\left\langle x y, x^{\prime} y^{\prime}\right\rangle-\frac{1}{4}\left(\left\langle x, x^{\prime}\right\rangle+\left\langle y, y^{\prime}\right\rangle+\left\langle z, z^{\prime}\right\rangle\right)^{2} .
\end{aligned}
$$

Clearly $(0, J, 0,0)$ and $\left(0, J_{1}, J_{1}, 0\right) \in X$.

5.7. $Y$ is the algebraic set cut out by the set of degree 3 polynomials $\left\{\partial f_{4} / \partial v: v \in V_{M}\right\}$. It contains the point $p(0, J, 0,0)\left(\right.$ resp. $\left.p\left(0,0, J^{\prime}, 0\right)\right)$ if and only if $J$ (resp. $J^{\prime}$ ) has rank at most 2 . Similarly $Z$ contains the point iff $J$ (resp. $J^{\prime}$ ) has rank 1 .

5.8. Let $\mathcal{K}=\mathbb{C}$, then the nontrivial outer automophism of $M=S L_{6}$ in Table $1\left(\mathrm{e}_{2}\right)$ acts on $V_{M}$ by sending $\left(\xi, J, J^{\prime}, \xi^{\prime}\right) \mapsto\left(\xi, \bar{J}, \overline{J^{\prime}}, \xi^{\prime}\right)$ where $\bar{J}$ and $\overline{J^{\prime}}$ denotes taking conjugation of the entries.

5.9. Let $G_{0}=\operatorname{Spin}(4,4)$ and by setting $\mathcal{J}$ to be the set of diagonal 3 by 3 complex matrices in $(25), V_{M}$ is the representation $\mathbb{C}^{2} \otimes \mathbb{C}^{2} \otimes \mathbb{C}^{2}$ of $M(\mathbb{C})=S L_{2}^{3}(\mathbb{C})$. We index this case by $s=0$.

\section{Dual pairs in $\widetilde{\boldsymbol{F}}_{4,4}$.}

6.1. In this section let $G=\widetilde{F}_{4,4}$. It has maximal compact subgroup $K^{\prime}=$ $S U_{2}(\widetilde{\alpha}) \times S p_{6}$. Let $G^{\prime}=\widetilde{\operatorname{Spin}}(5,4)$ and $G^{\prime \prime}=\widetilde{\operatorname{Spin}}(4,4)$. In this section we will prove Theorems 4.2 .1 and 4.3.1. We will retain the notations of $\S 4.2$ and $\S 4.3$.

6.2. The center $C$ of $\widetilde{\operatorname{Spin}}(4,4)$ is

$$
C:=\left\{\left(\epsilon_{1} \epsilon_{2} \epsilon_{3}, \epsilon_{1}, \epsilon_{2}, \epsilon_{3}\right) \in S U_{2}(\widetilde{\alpha}) \times S U_{2}^{3}: \epsilon_{i}= \pm 1 \in S U_{2}\right\} \simeq \mu_{2}^{3} .
$$

$(-1,-1,-1,-1) \in C$ is the nontrivial center of $\widetilde{F}_{4,4}$. We would like to interpret the subgroup $\widetilde{\operatorname{Spin}}(4,4)$ in $\widetilde{F}_{4,4}$ as the dual pair

$$
\widetilde{\operatorname{Spin}}(4,4) \times{ }_{C} C \text {. }
$$


We denote a character of $C$ by $\chi\left(s_{1}, s_{2}, s_{3}\right), s_{i} \in(\mathbb{Z} / 2 \mathbb{Z})$ such that

$$
\left(\epsilon_{1}, \epsilon_{2}, \epsilon_{3}\right) \mapsto \epsilon_{1}^{s_{1}} \epsilon_{2}^{s_{2}} \epsilon_{3}^{s_{3}} .
$$

Let $C^{\wedge}$ denote the character group of $C$.

The element $\epsilon_{1} \in C$ acts on $F_{4,4}$ by conjugation and it fixes the subgroup $\widetilde{\operatorname{Spin}}(5,4)$ in $\widetilde{F}_{4,4}$. It has maximal compact subgroup

$$
K=S U_{2}(\widetilde{\alpha}) \times\left(S U_{2}(A) \times \operatorname{Spin}(5)\right) .
$$

The center $C_{1} \subset C$ of $\widetilde{\operatorname{Spin}}(5,4)$ is the Klein 4 group

$$
C_{1}:=\left\{\left(\epsilon_{1}, \epsilon_{1}, \epsilon_{2}, \epsilon_{2}\right) \in C: \epsilon_{i}= \pm 1\right\} .
$$

We denote the character group of $C_{1}$ by $C_{1}^{\wedge}$. It consists of characters

$$
\chi\left(s_{1}, s_{2}\right):\left(\epsilon_{1}, \epsilon_{2}\right) \mapsto \epsilon_{1}^{s_{1}} \epsilon_{2}^{s_{2}}
$$

where $s_{i} \in \mathbb{Z} / 2 \mathbb{Z}$.

We have the following see-saw pairs in $\widetilde{F}_{4,4}$ :

$$
\begin{array}{lll}
\widetilde{\operatorname{Spin}}(5,4) & & C \\
\widetilde{\operatorname{Spin}}(4,4) & & C_{1} .
\end{array}
$$

6.3. The $S_{3}$ outer automorphism group on $\widetilde{\operatorname{Spin}}(4,4)$ permutes the 3 factor subgroups of $S U_{2}^{3} \subset M^{\prime \prime}$ and $G$ contains

$$
\left(\widetilde{\operatorname{Spin}}(4,4) \times_{C} C\right) \rtimes S_{3} .
$$

Hence if $\operatorname{Res}_{\operatorname{Spin}(4,4)}^{\widetilde{4}_{4,4}} \sigma(\mathbb{C}[k])$ contains $\sigma(\operatorname{Spin}(4,4), S(a, b, c)[k])$, then it will also contain

$$
{ }^{s} \sigma=\sigma\left(\operatorname{Spin}(4,4), S_{s(A)}^{a} \otimes S_{s(B)}^{b} \otimes S_{s(C)}^{c}[k]\right),
$$

where $s \in S_{3}$.

6.4. By (31) $\operatorname{Spin}(4,4) \rtimes S_{3} \subset F_{4,4}$ and $M^{\prime \prime}(\mathbb{C})=S L_{2}^{3}(\mathbb{C}) \rtimes S_{3}$. Set $\mathcal{K}=\mathbb{C}$ and we recall the definition of $\mathcal{J}_{i}$ and $W_{i}(i=1,2,3)$ in (26). The $W_{i}$ 's give the standard representations of each of the 3 factor groups of $S U_{2}^{3} \subset M^{\prime \prime}$. $V_{0}=W_{1} \oplus W_{2} \oplus W_{3}$. The outer automorphism group $S_{3}$ acts on $V_{0}$ by permuting the $W_{i}$ 's. Then $\mathbb{P} V_{0}$ has a dense $M^{\prime \prime}(\mathbb{C})$-orbit. There are two more orbits and their closures are

$$
\begin{aligned}
& X_{2}:=\mathbb{P}\left(W_{1} \oplus W_{2}\right) \cup \mathbb{P}\left(W_{2} \oplus W_{3}\right) \cup \mathbb{P}\left(W_{3} \oplus W_{1}\right) \\
& X_{1}:=\mathbb{P} W_{1} \cup \mathbb{P} W_{2} \cup \mathbb{P} W_{3} \subset X_{2} .
\end{aligned}
$$

The homogeneous ideal of $X_{2}$ in $\mathbb{P} V_{0}$ is generated by

$$
W_{0}=W_{1} \otimes W_{2} \otimes W_{3} \subset S^{3}\left(V_{0}\right)
$$


and it has coordinate ring

$$
A^{n}\left(X_{2}\right)=\sum_{a, b, c} S^{a}\left(W_{1}\right) \otimes S^{b}\left(W_{2}\right) \otimes S^{c}\left(W_{3}\right)
$$

where the sum is taken over all nonnegative integers $a, b, c$ such that $a+b+$ $c=n$ and $a b c=0$.

\section{Lemma 6.4.1.}

(a) $\mathbb{P} V_{0} \cap X=\mathbb{P} V_{0}$.

(b) $\mathbb{P} V_{0} \cap Y=X_{2}$.

(c) $\mathbb{P} V_{0} \cap Z$ is the empty set.

Proof. Let $J_{1}=(0,0,0 ; 0,0,1), J_{2}=(0,0,0 ; 0,1,1), J_{3}=(0,0,0 ; 1,1,1)$ and $v_{i}=\left(0, J_{i}, 0,0\right) \in X$ for $i=1,2,3 . p\left(v_{3}\right) \in\left(X \cap \mathbb{P} V_{0}\right) \backslash X_{2}$ so $\mathbb{P} V_{0} \cap X$ strictly contains $X_{2}$ and thus equals $\mathbb{P} V_{0}$. This proves (a). Note that $p\left(v_{3}\right) \notin Y$ so $\mathbb{P} V_{0} \cap Y \subset X_{2}$. On the other hand $p\left(v_{2}\right) \in Y \cap X_{2}$. This proves (b). Finally $p\left(v_{3}\right) \in X_{1}$ but it does not lie in $Z$. This proves (c).

6.5. Consider $\operatorname{Spin}(5,4) \subset F_{4,4}$ and $M^{\prime}=S U_{2} \times \operatorname{Spin}(5)=S U_{2} \times S p_{4}$. Then $V_{0}^{\prime}=W_{2} \oplus W_{3}$ gives the standard representation of $S p_{4}$. Note that $\mathbb{P} V_{0}^{\prime}$ is a single $S p_{4}$ orbit.

\section{Lemma 6.5.1.}

(a) $\mathbb{P} V_{0}^{\prime} \cap X=\mathbb{P} V_{0}^{\prime} \cap Y=\mathbb{P} V_{0}^{\prime}$.

(b) $\mathbb{P} V_{0}^{\prime} \cap Z$ is the empty set.

Proof. Since $V_{0}^{\prime} \subset V_{0}$, the lemma follows from Lemma 6.4.1.

6.6. Proof of Theorems 4.2.1(c)(d) and 4.3.1(c)(d). Part (d) follows easily from Proposition 3.3.1 since $S^{n} V_{0}^{\prime}=V_{0, n}$ and $S^{n} V_{0}=\sum_{a+b+c=n} S(a, b, c)$ respectively.

(c) By (20) we have

$$
\begin{aligned}
0 \rightarrow \operatorname{Res} \sigma_{X} \rightarrow \sum_{n=0}^{\infty} \mathbf{H}\left(\operatorname{Spin}(5,4), V_{0, n}[d-1+n]\right) \\
\\
\rightarrow \sum_{n=0}^{\infty} \mathbf{H}\left(\operatorname{Spin}(5,4), V_{0, n}[d+3+n]\right) \rightarrow 0 .
\end{aligned}
$$

Considering the infinitesimal characters of the summands gives (13).

The restriction of $\sigma_{X}$ to Spin $(4,4)$ and (14) have been proven in Prop. 4.6.1 of [L2]. This proves (c).

Proof of Theorem 4.2.1(b). $r_{3}: V_{M} \rightarrow S^{3}\left(V_{0}^{\prime}\right)$ and since the codomain is irreducible, $r_{3}$ is either the zero map or a surjection. However the image of $r_{3}$ has to cut out the empty set so $r_{3}=0$. This implies $r_{n}=0$ and $R_{n}=S^{n}\left(V_{0}\right)$ for all $n$. 
6.7. Proof of Theorem 4.3.1(b). $r_{3}: V_{M} \rightarrow S^{3}\left(V_{0}\right)$ and

$$
\begin{aligned}
V_{M} & =S_{A}^{1}+S_{B}^{1}+S_{C}^{1}+\left(S_{A}^{1} \otimes S_{B}^{1} \otimes S_{C}^{1}\right) \\
S^{3}\left(V_{0}\right) & =\sum_{a+b+c=3} S(a, b, c)
\end{aligned}
$$

as representations of $M^{\prime \prime}$. Thus image of $r_{3}$ is either 0 or $W_{0}$ (cf. (32)). By Lemma 6.4.1 the image has to cut out $X_{2}$ so it is $W_{0}$. Moreover $W_{0}$ generates the ideal of $X_{2}$ and thus $R_{n}=A^{n}\left(X_{2}\right)$.

6.8. Recall that the minimal representation $\sigma_{Z}$ of $\widetilde{F}_{4,4}$ has $K$-types

$$
\sum_{n=0}^{\infty} S_{\widetilde{\alpha}}^{n+1}\left(\mathbb{C}^{2}\right) \otimes \pi_{S p_{6}}\left(n \varpi_{3}\right) .
$$

Suppose

$$
\operatorname{Res} \frac{\widetilde{F}_{4,4}}{\operatorname{Spin}(5,4) \times C_{1}} \sigma_{Z}=\sum_{\chi \in C_{1}^{\wedge}} \Theta^{\prime}(\chi) \otimes \chi .
$$

The center of $\widetilde{F}_{4,4}$ acts nontrivially on $\sigma_{Z}$ and hence $C_{1}$ only acts by the characters $\chi(1,0)$ and $\chi(1,1)$. Theorem $4.2 .1(\mathrm{a})$ is a consequence of the following theorem:

Theorem 6.8.1. Let $\epsilon=0,1$. Then $\Theta^{\prime}(\chi(1, \epsilon))=\sigma\left(\widetilde{\operatorname{Spin}}(5,4), V_{0, \epsilon}[4]\right)$ and it has $K$-types $\left(K=S U_{2}(\widetilde{\alpha}) \times_{\mu_{2}}\left(S U_{2} \times \operatorname{Spin}(5)\right)\right)$

$$
\sum_{a, b \geq 0} S_{\widetilde{\alpha}}^{a+2 b+1+\epsilon}\left(\mathbb{C}^{2}\right) \otimes S^{a}\left(\mathbb{C}^{2}\right) \otimes V_{a, 2 b+\epsilon} .
$$

Proof. By Lemma 6.5.1, $\mathcal{O}^{\prime}$ is empty so $r_{2}^{\prime}: S^{2} V_{0}^{\prime} \rightarrow S^{2} V_{0}^{\prime}$ is not the zero map. Hence $r_{2}^{\prime}$ is a surjection and Theorem 3.5.1(a) applies. The following lemma proves the claim about the $K$-types and completes the proof of the theorem:

\section{Lemma 6.8.2.}

$$
\operatorname{Res}_{S U_{2} \times \operatorname{Spin}(5) \times C^{\prime}}^{S p_{6}} \pi\left(n \varpi_{3}\right)=\sum_{a+b=n} S^{a}(\mathbb{C}) \otimes \pi_{\operatorname{Spin}(5)}\left(a \varpi_{1}+b \varpi_{2}\right) \otimes \chi(a, b) .
$$

We omit the proof of the lemma since branching law of $S p_{6}$ is known (see Equation (25.27) $[\mathbf{F H}])$.

6.9. Suppose

$$
\operatorname{Res} \frac{\widetilde{F}_{4,4}}{\operatorname{Spin}(4,4) \times C} \sigma_{Z}=\sum_{\chi \in C^{\wedge}} \Theta(\chi) \otimes \chi .
$$

Since the center of $\widetilde{F}_{4,4}$ acts nontrivially on $\sigma_{Z}, C$ can only act by the characters $\chi\left(s_{1}, s_{2}, s_{3}\right)$ where $s_{1}+s_{2}+s_{3}$ is an odd integer.

Theorem 4.5.1(a) is a consequence of the following theorem: 


\section{Theorem 6.9.1.}

(a) $\Theta(\chi(1,1,1))=\sigma(\widetilde{\operatorname{Spin}}(4,4), \mathbb{C}[3])$.

(b) $\Theta(\chi(1,0,0))=\sigma(\widehat{\operatorname{Spin}}(4,4), S(1,0,0)[4])$.

(c) $\Theta(\chi(0,1,0))=\sigma(\widetilde{\operatorname{Spin}}(4,4), S(0,1,0)[4])$.

(d) $\Theta(\chi(0,0,1))=\sigma(\widehat{\operatorname{Spin}}(4,4), S(0,0,1)[4])$.

$\Theta(\chi(1,1,1))$ (resp. $\Theta(\chi(1,0,0)))$ has $K$-types $\left(K=S U_{2} \times S U_{2}^{3}\right)$

$$
\sum_{n, a, b, c \geq 0} S_{\widetilde{\alpha}}^{1+n}(\mathbb{C}) \otimes S(a, b, c)
$$

where the sum is taken over all nonnegative integers $n, a, b, c$ such that $a+$ $b+c \geq n, a+b-c \leq n, a-b+c \leq n, b+c-a \leq n$ and $n \equiv a \equiv b \equiv c$ $(\bmod 2)($ resp. $n \equiv a \not \equiv b \equiv c(\bmod 2))$.

The $K$-types of $\Theta(\chi(0,1,0))$ and $\Theta(\chi(0,0,1))$ differ from that of $\Theta(\chi(1$, $0,0))$ by permuting $a, b, c$ in (34) accordingly under the action of $S_{3}(c f . \S 6.3)$.

Proof. By the action of $S_{3}$, it suffices to prove (a) and (b). By the see-saw pair (30), we note that

$$
\Theta^{\prime}(\chi(1,0))=\Theta(\chi(1,1,1))+\Theta(\chi(1,0,0)) .
$$

The branching rule from $\operatorname{Spin}(5)$ to $\operatorname{Spin}(4)=S U_{2}^{2}$ is well-known (see eqn (25.34) $[\mathbf{F H}]$ ). Applying this to Theorem 6.8.1 shows that the sum of the $K$-types of (a) and (b) agrees with the $K$-types in (35). A $K$-type

$$
S^{n}\left(\mathbb{C}^{2}\right) \otimes S(a, b, c)
$$

in (35) will belong to $\Theta(\chi(1,1,1))$ (resp. $\Theta(\chi(1,0,0)))$ if and only if $a-b$ is an even (resp. odd) integer. This proves (34).

By naturality, the composition of the map

$$
I^{2}(Z) \stackrel{r_{2}}{\rightarrow} S^{2} V_{0} \rightarrow S^{2} V_{0}^{\prime}=S_{B}^{2}+S_{C}^{2}+\mathbb{C}_{B} \otimes \mathbb{C}_{C}
$$

is the map $r_{2}^{\prime}$ in the proof of Theorem 6.8 .1 and it is surjective. This implies that the image contains $S_{B}^{2}+S_{C}^{2}+\mathbb{C}_{B} \otimes \mathbb{C}_{C}$. Since $r_{2}$ commutes with the action of $S_{3}$ (cf. $\S 6.3$ ), $r_{2}$ is surjective and the theorem follows from Theorem 3.5.1(c).

\section{Dual pairs in $E_{6,4}$.}

7.1. Let $G^{\prime} \subset G$ be one of the dual pairs (5) to (7). We set $s=2,4,8$ and we define

$$
\mathcal{J}_{s}^{p}=\left\{\left(0,0,0 ; x_{1}, x_{2}, x_{3}\right) \in \mathcal{J}\left(\mathcal{K}_{s}\right): x_{i} \in \mathcal{K}_{s},\left\langle x_{i}, z\right\rangle=0 \text { for all } z \in \mathcal{K}_{s / 2}\right\}
$$

then

$$
V_{0}=\left\{\left(0, J, J^{\prime}, 0\right) \in V_{M}: J, J^{\prime} \in \mathcal{J}_{s}^{p}\right\}
$$


It is known that $M^{\prime}(\mathbb{C})$ has a dense orbit in $\mathbb{P} V_{0}[\mathbf{S K}]$. The orbits and their coordinate rings have been extensively studied and they are documented in $\S 7$ [GW2]. We will make use of these results to determine $R_{\bullet}$.

7.2. Consider $G=\widetilde{E}_{6,4} \rtimes \mathbb{Z} / 2 \mathbb{Z} \supset G^{\prime}=\widetilde{F}_{4,4} \times \mathbb{Z} / 2 \mathbb{Z}$. Set $s=2$. Then $V_{0}=\mathbb{C}^{6}$ in $(36)$ is the standard representation of $M^{\prime}(\mathbb{C})=S p_{6}(\mathbb{C}) \times \mathbb{Z} / 2 \mathbb{Z}$. It is well-known that $\mathbb{P} V_{0}$ is a single orbit of $M^{\prime}(\mathbb{C})$.

\section{Lemma 7.2.1.}

(a) $\mathbb{P} V_{0} \cap X=\mathbb{P} V_{0} \cap Y=\mathbb{P} V_{0}$.

(b) $\mathbb{P} V_{0} \cap Z$ is an empty set.

Proof. (a) It suffices to show that $\mathbb{P} V_{0} \cap Y$ is nonempty since $X \supset Y$ and $\mathbb{P} V_{0}$ is a single orbit of $M^{\prime}(\mathbb{C})$. Let $J=(0,0,0 ; s, 0,0) \in \mathcal{J}\left(\mathcal{K}_{2}\right)$ where $s=\operatorname{diag}(1,-1) \in \mathcal{K}_{2}$. By $(36)(0, J, 0,0) \in V_{0}$. Since $\operatorname{det}(J)=0, p(J) \in Y$ by $\S 5.7$.

(b) We will prove this by contradiction. Suppose on the contrary $\mathbb{P} V_{0} \cap Z$ is nonempty and it equals $\mathbb{P} V_{0}$. Let $v=\left(0, J, J^{\prime}, 0\right) \in V_{0}$ as given in (36) and assume that $J \neq 0$. Since $p(v) \in Z$, by Lemma 5.5.1 and (27) $J$ has rank at most 1 . By (24), $J=0$. This yields the contradiction.

Proof of Theorem 4.5.1. (a) By Lemma 7.2.1(b), $r_{2}: \mathfrak{s l}_{6} \rightarrow S^{2}\left(\mathbb{C}^{6}\right)$ is nonzero. Since the image is irreducible $r_{2}$ is a surjection. (a) follows from Theorem 3.5.1(c).

(b) and (c) follow from Lemma 7.2.1(a) and Theorem 3.5.1(a) since $R_{n}=$ $S^{n}\left(\mathbb{C}^{6}\right)$.

By (20) we have

$$
\begin{aligned}
0 \rightarrow \operatorname{Res} \sigma_{X} \rightarrow \sum_{n=0}^{\infty} \mathbf{H}\left(\widetilde{F}_{4,4}, S^{n}\right. & \left.\left(\mathbb{C}^{6}\right)[9+n]\right) \otimes \chi^{n} \\
& \rightarrow \sum_{n=0}^{\infty} \mathbf{H}\left(\widetilde{F}_{4,4}, S^{n}\left(\mathbb{C}^{6}\right)[13+n]\right) \otimes \chi^{n} \rightarrow 0 .
\end{aligned}
$$

Finally considering the infinitesimal characters of the above summands gives (15).

7.3. Consider $G_{0}=E_{6,4} \rtimes \mathbb{Z} / 2 \mathbb{Z} \supset G_{0}^{\prime \prime}=\operatorname{Spin}(4,4) \times_{\mu_{2}^{2}}\left(U_{1}^{2} \rtimes \mathbb{Z} / 2 \mathbb{Z}\right)$ and $M^{\prime \prime}=S U_{2}^{3} \times_{\mu_{2}^{2}}\left(U_{1}^{2} \rtimes \mathbb{Z} / 2 \mathbb{Z}\right)$. Here we identify $U_{1}^{2}=\left\{\left(t_{1}, t_{2}, t_{3}\right): t_{i} \in\right.$ $\left.U_{1}, t_{1} t_{2} t_{3}=1\right\}$.

Let $\chi_{0}\left(a_{1}, a_{2}, a_{3}\right):\left(t_{1}, t_{2}, t_{3}\right) \mapsto t_{1}^{a_{1}} t_{2}^{a_{2}} t_{3}^{a_{3}}$ be a character of $U_{1}^{2}$ where $a_{i} \in$ $\mathbb{Z}$. Let $\chi\left(a_{1}, a_{2}, a_{3}\right)$ be the unique irreducible representation of $U_{1}^{2} \rtimes \mathbb{Z} / 2 \mathbb{Z}$ containing $\chi_{0}\left(a_{1}, a_{2}, a_{3}\right)$. Note that $\chi(a, a, a)=\mathbb{C}$ and if not all the $a_{i}$ 's are the same, then $\chi\left(a_{1}, a_{2}, a_{3}\right)=\chi_{0}\left(a_{1}, a_{2}, a_{3}\right)+\chi_{0}\left(-a_{1},-a_{2},-a_{3}\right)$. Clearly 
$\chi\left(a_{1}, a_{2}, a_{3}\right)=\chi\left(a_{1}-a, a_{2}-a, a_{3}-a\right)=\chi\left(-a_{1},-a_{2},-a_{3}\right)$. Therefore we may assume that $\left(a_{1}, a_{2}, a_{3}\right)$ takes values from the set

$$
\begin{aligned}
T:=\left\{\left(a_{1}, a_{2}, a_{3}\right) \in \mathbb{Z}^{3}: a_{i}>a_{i+1}=0\right. & \geq a_{i+2} \\
& \text { for some } i=1,2,3\} \cup\{(0,0,0)\} .
\end{aligned}
$$

Set $\mathcal{K}=\mathcal{K}_{2}$ and we define $\mathcal{J}_{i}$ and $W_{i}(i=1,2,3)$ using (26). Then $V_{0}=W_{1} \oplus W_{2} \oplus W_{3} . W_{i}=W_{i 1} \oplus W_{i 2}$ where $W_{i 1}$ and $W_{i 2}$ are the standard representations of $S U_{2}$. $\left(t_{1}, t_{2}, t_{3}\right) \in U_{1}^{2}$ acts on $W_{i 1}\left(\right.$ resp. $\left.W_{i 2}\right)$ by multiplication by $t_{i}$ (resp. $\left.t_{i}^{-1}\right)$.

Write $V_{0}=\sum_{i, j} W_{i j}$ and we denote an element of $V_{0}$ by $\left(w_{i j}\right)$ where $w_{i j} \in W_{i j}$. Let $\mathcal{V}$ denote the subset of $V_{0}$ consisting of $\left(w_{i j}\right)$ satisfying the following:

1. For each $j=1,2$, at least 2 of $w_{1 j}, w_{2 j}, w_{3 j}$ is zero.

2. For each $i=1,2,3$, either $w_{i 1}=0$ or $w_{i 2}=0$.

Then one can show that $Z \cap \mathbb{P} V_{0}=\mathbb{P} \mathcal{V}$ and the ideal of $\mathbb{P} \mathcal{V}$ is generated by degree 2 polynomials. Its coordinate ring is

$$
A^{n}(\mathbb{P} \mathcal{V})=\sum S\left(\left|a_{1}\right|,\left|a_{2}\right|,\left|a_{3}\right|\right) \otimes \chi\left(a_{1}, a_{2}, a_{3}\right)
$$

where the sum is taken over all $\left(a_{1}, a_{2}, a_{3}\right) \in T$ satisfying $\left|a_{1}\right|+\left|a_{2}\right|+\left|a_{3}\right|=n$. By Theorem 3.5.1(c) we get the dual pair correspondence of $G^{\prime \prime}$ in $G$.

\section{Theorem 7.3.1.}

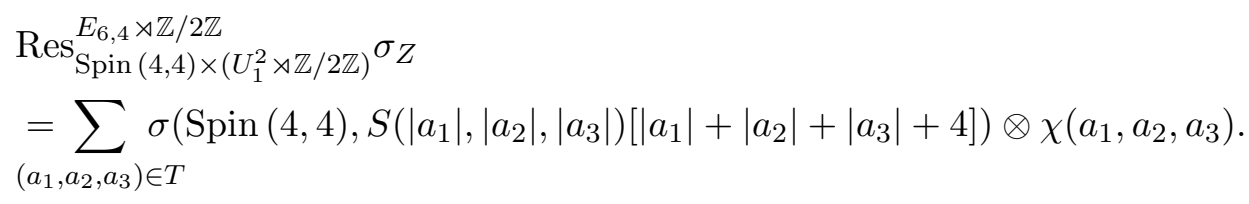

The $K$-types of the summands could be calculated using (12) by applying the branching rule from $M=S U_{6}$ to $S U_{2}^{3} \times U_{1}^{2} \subset M^{\prime \prime}$. This in turn could be computed using the Littlewood-Richardson rule (see [FH, p. 456]).

Note that the summands of the above correspondence also appear in Theorem 4.3.1(b). This follows from the fact that the dual pairs $G_{0}^{\prime \prime}$ and $F_{4,4} \times \mathbb{Z} / 2 \mathbb{Z}$ form a see-saw pair in $G_{0}$. Similarly restrictions of $\sigma_{Y}$ and $\sigma_{X}$ to $G_{0}^{\prime \prime}$ will yield representations of $\widehat{\operatorname{Spin}}(4,4)$ appearing in Theorem 4.3.1(c) and $(\mathrm{d})$.

\section{Dual pair in $E_{7,4}$.}

8.1. Consider $G_{0}=E_{7,4} \supset G_{0}^{\prime}=\left(E_{6,4} \times_{\mu_{3}} U_{1}\right) \rtimes \mathbb{Z} / 2 \mathbb{Z}$ and $M^{\prime}(\mathbb{C})=$ $G L_{6} \rtimes \mathbb{Z} / 2 \mathbb{Z}$. The nontrivial element in $\mathbb{Z} / 2 \mathbb{Z}$ acts on $E_{6,4}$ as the outer automorphism. It also acts on $U_{1}$ by sending $z \mapsto z^{-1}$.

Set $s=4$ and define $V_{0}$ as in $(36) . z \in U_{1}(\mathbb{C})=\mathbb{C}^{*}$ will act on $V_{0}$ by

$$
\left(0, J, J^{\prime}, 0\right) \rightarrow\left(0, z J, z^{-1} J^{\prime}, 0\right) .
$$


Recall $\mathcal{K}_{4}=M_{2}(\mathbb{C})$ and let $\mathcal{K}_{u}\left(\right.$ resp. $\left.\mathcal{K}_{l}\right)$ denote the subspace of strictly upper (resp. lower) triangular matrices. Define

$$
\begin{aligned}
\mathcal{J}_{u} & =\left\{\left(0,0,0 ; c_{1}, c_{2}, c_{3}\right) \in \mathcal{J}\left(\mathcal{K}_{4}\right): c_{i} \in \mathcal{K}_{u}\right\} \\
V_{u} & =\left\{\left(0, J, J^{\prime}, 0\right) \in V_{0}: J, J^{\prime} \in \mathcal{J}_{u}\right\} .
\end{aligned}
$$

Similarly we define $\mathcal{J}_{l}$ and $V_{l}$ by replacing $\mathcal{K}_{u}$ with $\mathcal{K}_{l} . V_{u}$ (resp. $V_{l}$ ) gives the standard (resp. dual) representation of $G L_{6}$. Thus $V_{0}=\mathbb{C}^{6} \oplus\left(\mathbb{C}^{6}\right)^{*}$ as a representation of $M^{\prime}(\mathbb{C})=G L_{6} \rtimes \mathbb{Z} / 2 \mathbb{Z}$.

There are only two nontrivial proper orbits of $M^{\prime}$ in $\mathbb{P} V_{0}$ (cf. $\S 6$ [GW2]). Their closures are

$$
\begin{aligned}
X_{1} & =\mathbb{P} \mathbb{C}^{6} \cup \mathbb{P}\left(\mathbb{C}^{6}\right)^{*} \\
\mathcal{F} & =\left\{\left(v, v^{*}\right) \in \mathbb{P} V_{0}: f_{0}:=\left\langle v, v^{*}\right\rangle=0\right\} .
\end{aligned}
$$

Note that $X_{1} \subset \mathcal{F}$. The inner product $f_{0}$ defining $\mathcal{F}$ is an $M^{\prime}$-invariant quadratic form in $S^{2} V_{0}$ and this in turn induces an inclusion

$$
S^{n} V_{0} \cdot f_{0} \hookrightarrow S^{n+2} V_{0} .
$$

The coordinate ring $A^{n}(\mathcal{F})$ is the quotient of the above inclusion. Recall $\S 4.7$ where we define $V_{a, b}=\pi_{S L_{6}}\left(a \varpi_{1}+b \varpi_{6}\right)$. Then

$$
\begin{aligned}
A^{n}(\mathcal{F}) & =\sum_{a+b=n} V_{a, b} \otimes \chi_{1}^{a-b} \\
A^{n}\left(\mathbb{P} V_{0}\right) & =A^{n}(\mathcal{F})\left[f_{0}\right]=\sum_{a+b+2 m=n} V_{a, b} \otimes \chi_{1}^{a-b}
\end{aligned}
$$

as a representation of $G L_{6}=S L_{6} \times_{\mu_{6}} U_{1}$.

Lemma 8.1.1.

(a) $\mathbb{P} V_{0} \cap Z=\mathbb{P} \mathbb{C}^{6} \cup \mathbb{P}\left(\mathbb{C}^{6}\right)^{*}$.

(b) $\mathcal{F}=\mathbb{P} V_{0} \cap X=\mathbb{P} V_{0} \cap Y$.

Proof. Define

$$
\begin{gathered}
s=\left(\begin{array}{ll}
0 & 1 \\
0 & 0
\end{array}\right), \quad t=\left(\begin{array}{cc}
0 & 0 \\
1 & 0
\end{array}\right) \in \mathcal{K}_{4}=M_{2}(\mathbb{C}), \\
x_{1}=(0,0,0 ; s, 0,0), \quad x_{2}=(0,0,0 ; t, 0,0) \in \mathcal{J}\left(\mathcal{K}_{4}\right), \\
T_{1}=\left(0, x_{1}, 0,0\right), \quad T_{2}=\left(0, x_{1}+x_{2}, 0,0\right), \quad T_{3}=\left(0, x_{1}, x_{2}, 0\right) \in V_{0} .
\end{gathered}
$$

By Lemma 5.5.1 $p\left(T_{1}\right) \in Z \cap \mathbb{P} V_{0}$. Hence $Z \cap \mathbb{P} V_{0}$ is nontrivial and it must contain $X_{1} . x_{1}+x_{2}$ has rank 2 so $p\left(T_{2}\right) \in Y \backslash Z$ (cf. $\S 5.7$ ) and $p\left(T_{2}\right) \notin X_{1}$. This proves (a) and implies that $\mathbb{P} V_{0} \cap Y \supseteq \mathcal{F}$.

Next $p\left(T_{3}\right) \in \mathbb{P} V_{0} \backslash \mathcal{F}$. $f_{4}\left(T_{3}\right) \neq 0$ so $T_{3} \notin X$. Since $\mathcal{F}$ is maximal proper, $\mathcal{F} \supseteq X \cap \mathbb{P} V_{0} \supseteq Y \cap \mathbb{P} V_{0}$. This proves (b). 
Proof of Theorem 4.7.1. (a) The image of $r_{2}$ must be cut out $X_{1}$ so it is either $V_{1,1}$ or $\mathbb{C} f_{0}+V_{1,1}$ in $S^{2} V_{0}$.

We claim that the image cannot be $V_{1,1}$. Indeed if otherwise by Theorem 3.5.1(b) the restriction of $\sigma_{Z}$ will contain $\sigma^{\prime}=\sigma\left(E_{6,4}, \mathbb{C}[6]\right) . \sigma^{\prime}$ contains the lowest $K^{\prime}$-type $\tau=S_{\widetilde{\alpha}}^{4}\left(\mathbb{C}^{2}\right) \otimes \mathbb{C}\left(K^{\prime}=S U_{2}(\widetilde{\alpha}) \times S U_{6}\right)$. Therefore $\tau$ is a subrepresentation of the $K$-type

$$
S_{\widetilde{\alpha}}^{4}\left(\mathbb{C}^{2}\right) \otimes \pi_{\text {Spin (12) }}\left(2 \varpi_{6}\right)
$$

in $\sigma_{Z}$. However the tables of $[\mathbf{K P}]$ show that the above does not contain $\tau$. This proves the claim.

Finally since $V_{1,1}+\mathbb{C} f_{0}$ generates the homogeneous ideal of $X_{1}$ so $R_{\bullet}=$ $A^{\bullet}(\mathcal{F})$ (cf. (37)). This proves (a).

(b) Similar to (a) the image of $r_{3}$ cuts out $\mathcal{F}$ and it has to be $V_{0} \cdot f_{0} \subset$ $S^{3} V_{0}$. The ideal generated by $V_{0} f_{0}$ contains all homogeneous polynomials vanishing on $\mathcal{F}$ except $\mathbb{C} f_{0}$. Thus $R_{\bullet}=A^{\bullet}(\mathcal{F})+\mathbb{C} f_{0}$. Finally we note that $\mathbf{H}\left(E_{6,4}, \mathbb{C}[12]\right)$ is irreducible (cf. $\left.\S 3.3\right)$.

(c) By (20) we get

$$
\begin{aligned}
0 \rightarrow \operatorname{Res} \sigma_{X} \rightarrow \sum_{a, b, n} \mathbf{H}\left(\widetilde{E}_{6,4}, V_{a, b}[15+2 n+a+b]\right) \otimes \chi_{1}^{a-b} & \\
& \rightarrow \sum_{a, b, n} \mathbf{H}\left(\widetilde{E}_{6,4}, V_{a, b}[19+2 n+a+b]\right) \otimes \chi_{1}^{a-b} \rightarrow 0
\end{aligned}
$$

The summands on the right are all irreducible and unitary, and they also appear as summands in the middle term. Therefore removing these representations from (38) gives

$$
0 \rightarrow \operatorname{Res} \sigma_{X} \rightarrow \sum_{n=0,1} \sum_{a, b} \mathbf{H}\left(\widetilde{E}_{6,4}, V_{a, b}[15+2 n+a+b]\right) \otimes \chi_{1}^{a-b} \rightarrow 0
$$

This completes the proof of (c).

Note that in (c) the image of $r_{4}$ is one dimensional and it cuts out $\mathcal{F}$ and it has to be $\left(f_{0}\right)^{2} \subset S^{4} V_{0}$. Therefore $R_{\bullet}$ is not reduced. This example shows that the containment in Theorem 3.5.1(b) may be proper. 


\section{Dual pairs in $E_{8,4}$.}

9.1. In this section we consider $\widetilde{E}_{8,4} \supset \widetilde{E}_{7,4} \times_{\mu_{3}} S U_{2}$ and $M^{\prime}=\operatorname{Spin}(12) \times$ $S U_{2}$. Set $s=8$ and define $V_{0}$ by (36).

9.2. Recall $U_{0}$ in $\S 5.3$. Define $U_{e}:=\mathbb{C}_{2} \oplus \mathbb{C} e_{3} \subset U_{0}$. By (22) $S L\left(U_{e}\right)$ acts on $\mathcal{K}_{8}$. This induces an action of $S L\left(U_{e}\right)$ on $\left(0, J, J^{\prime}, 0\right) \in V_{0}$ where $S L\left(U_{e}\right)$ acts uniformly on each of the nonzero entries of $J$ and $J^{\prime}$.

For $i=1,2$, define $J_{i}=\left(0,0,0 ; x_{i 1}, x_{i 2}, x_{i 3}\right) \in \mathcal{J}$ where $x_{i j}=\left(0,0 ; w_{i j 1} e_{2}\right.$, $\left.-w_{i j 2} e_{3}\right)$ in $\mathcal{K}_{8}($ cf. $(21))$ and $w_{i j k} \in \mathbb{C}$. Let $v=\left(0, J_{1}, J_{2}, 0\right) \in V_{0}$. Denote the set of such vectors $v$ in $V_{0}$ by $V_{1}$.

Similarly for $i=1,2$, define $J_{i}^{\prime}=\left(0,0,0 ; x_{i 1}^{\prime}, x_{i 2}^{\prime}, x_{i 3}^{\prime}\right) \in \mathcal{J}$ where $x_{i j}^{\prime}=$ $\left(0,0 ; w_{i j 1}^{\prime} e_{3}, w_{i j 2}^{\prime} e_{2}\right) \in \mathcal{K}_{8}$ and $w_{i j k}^{\prime} \in \mathbb{C}$. Let $v^{\prime}=\left(0, J_{1}^{\prime}, J_{2}^{\prime}, 0\right) \in V_{0}$. Denote the set of such vectors $v^{\prime}$ in $V_{0}$ by $V_{2}$.

Note that $V_{0}=V_{1} \oplus V_{2}$. Both $V_{1}$ and $V_{2}$ give the standard representations of $\operatorname{Spin}(12)$ in $M(\mathbb{C})$. The invariant quadratic forms on $V_{1}$ and $V_{2}$ are given respectively by

$$
\begin{aligned}
& q_{1}\left(w_{i j k}\right)=\sum_{j=1}^{3}\left(w_{1 j 1} w_{2 j 2}-w_{1 j 2} w_{2 j 1}\right) \\
& q_{2}\left(w_{i j k}^{\prime}\right)=\sum_{j=1}^{3}\left(w_{1 j 1}^{\prime} w_{2 j 2}^{\prime}-w_{1 j 2}^{\prime} w_{2 j 1}^{\prime}\right) .
\end{aligned}
$$

Let $\omega=\left(\begin{array}{cc}0 & 1 \\ -1 & 0\end{array}\right) \in S L\left(U_{e}\right),\left(w_{i j k}\right) \in V_{1}$ and $\left(w_{i j k}^{\prime}\right) \in V_{2}$. The action of $\omega$ on $V_{0}$ commutes with that of Spin(12) and $\omega V_{2}=V_{1}$ by sending $\left(w_{i j k}^{\prime}\right) \mapsto\left(w_{i j k}\right)$ where $w_{i j k}=w_{i j k}^{\prime}$. Let $w_{1}, w_{2} \in V_{1}$, define $\phi: V_{1} \otimes U_{e} \rightarrow V_{0}$ by

$$
\phi: w_{1} \otimes e_{2}+w_{2} \otimes e_{3} \rightarrow w_{1}+\omega^{-1} w_{2} .
$$

Then $\phi$ is an isomorphism of representations of $M^{\prime}(\mathbb{C})=\operatorname{Spin}(12) \times S L\left(U_{e}\right)$.

9.3. We will describe the orbits of $M^{\prime}(\mathbb{C})$ on $\mathbb{P} V_{0} . \mathbb{P} V_{0}$ has a dense $M^{\prime}(\mathbb{C})$ orbit. It contains four additional orbits and we denote their closures by $X_{1}$, $Y_{1}, Y_{2}, Z_{1}$. Let $\langle$,$\rangle denote the inner product induced by quadratic form q_{1}$ on $V_{1}$ and let $v=w_{1} \otimes e_{2}+w_{2} \otimes e_{2}$. Then $X_{1}$ is a hypersurface whose ideal is generated by a degree 4 polynomial $f_{4}^{\prime}$

$$
f_{4}^{\prime}(v)=\operatorname{det}\left(\begin{array}{cc}
\left\langle w_{1}, w_{1}\right\rangle & \left\langle w_{1}, w_{2}\right\rangle \\
\left\langle w_{2}, w_{1}\right\rangle & \left\langle w_{2}, w_{2}\right\rangle
\end{array}\right)
$$

$Y_{1}$ is the complete intersection of the 3 quadrics

$$
\left\langle w_{1}, w_{1}\right\rangle=\left\langle w_{1}, w_{2}\right\rangle=\left\langle w_{2}, w_{2}\right\rangle=0 .
$$


$Y_{2} \subset X_{1}$ is the subvariety $\mathbb{P} W \times \mathbb{P} U$. Let $Q \subset \mathbb{P} W$ be defined by $\left\langle w_{1}, w_{1}\right\rangle=0$. Then $Z_{1}=Q \times \mathbb{P} U=Y_{1} \cap Y_{2}$ is the unique minimal closed orbit in $\mathbb{P} V_{0}$. $Y_{1} \cup Y_{2}$ is cut out by cubics

$$
\begin{aligned}
& f_{a}(v):=\operatorname{det}\left(\begin{array}{cc}
\left\langle w_{1}, w_{1}\right\rangle & \left\langle w_{1}, a\right\rangle \\
\left\langle w_{2}, w_{1}\right\rangle & \left\langle w_{2}, a\right\rangle
\end{array}\right)=0, \\
& f_{b}^{\prime}(v):=\operatorname{det}\left(\begin{array}{cc}
\left\langle b, w_{1}\right\rangle & \left\langle b, w_{2}\right\rangle \\
\left\langle w_{2}, w_{1}\right\rangle & \left\langle w_{2}, w_{2}\right\rangle
\end{array}\right)=0
\end{aligned}
$$

for all $a, b \in W$. Let $I_{0}$ be the homogeneous ideal generated by $\left\{f_{a}, f_{b}^{\prime}\right.$ : $a, b \in W\}$. We claim that $I_{0}$ is the homogeneous ideal of $Y_{1} \cup Y_{2}$. Indeed suppose $f$ vanishes on $Y_{1} \cup Y_{2}$, by (39) we may assume that modulo $I_{0}$

$$
f\left(w_{1}, w_{2}\right)=h_{1}\left(w_{1}\right)\left\langle w_{1}, w_{1}\right\rangle+h_{2}\left(w_{1}, w_{2}\right)\left\langle w_{1}, w_{2}\right\rangle+h_{3}\left(w_{2}\right)\left\langle w_{2}, w_{2}\right\rangle
$$

where $h_{1}, h_{2}, h_{3}$ are polynomials on $V_{1}, W$ and $V_{2}$ respectively. Since $f\left(w_{1}\right.$, $\left.w_{2}\right)=0$ whenever $w_{1}$ is parallel to $w_{2}$, we get $h_{1}=h_{3}=0$ and $h_{2}$ vanishes on $Y_{2}$. Thus $f \in I_{0}$ and this proves the claim.

Recall $\S 4.8$ where $V_{a, b}=\pi_{\text {Spin (12) }}\left(a \varpi_{1}+b \varpi_{2}\right)$. Then the coordinate rings are:

$$
\begin{aligned}
A^{\bullet}\left(\mathbb{P} V_{0}\right)= & A^{\bullet}\left(X_{1}\right)\left[f_{4}^{\prime}\right] \\
A^{n}\left(X_{1}\right)= & \sum^{*} V_{a+2 d, c} \otimes S^{a+2 b}(U) \\
& \text { where the sum } \sum^{*} \text { is taken over } a, b, c, d \text { satisfying (16). }
\end{aligned}
$$

$$
A^{n}\left(Y_{1} \cup Y_{2}\right)=\sum_{a+2 b+2 c=n, b c=0} V_{a, c} \otimes S^{a+2 b}(U) .
$$

The coordinate rings except (44) are given in $\S 5, \S 6$ [GW2]. Since $A^{n}\left(Y_{1} \cup\right.$ $\left.Y_{2}\right)$ is a quotient of $A^{n}\left(X_{1}\right)$ which is multiplicity free, (44) follows from (42) and (43).

\section{Lemma 9.3.1.}

(a) $X \cap \mathbb{P} V_{0}=X_{1}$.

(b) $Y \cap \mathbb{P} V_{0}=Y_{1} \cup Y_{2}$.

(c) $Z \cap \mathbb{P} V_{0}=Z_{1}$.

Proof. For the ease of notations, suppose $J_{i}=\left(0,0,0 ; c_{i 1}, c_{i 2}, c_{i 3}\right) \in \mathcal{J}(i$ $=1,2)$, then we denote $\left(0, J_{1}, J_{2}, 0\right) \in V_{0}$ by $\left(c_{11}, c_{12}, c_{13} \mid c_{11}, c_{12}, c_{13}\right)$. For $i=2,3$, let $x_{i}=\left(0,0 ; e_{i}, e_{i}\right), y_{i}=\left(0,0 ; e_{i}, 0\right) \in \mathcal{K}_{8}$ and (cf. $\left.(21)\right)$. 
(a) Set $v_{1}:=\left(x_{2}, x_{2}, x_{2} \mid 0,0,0\right) \in V_{0}$. Then $f_{4}\left(v_{1}\right) \neq 0$. Hence $X \cap \mathbb{P} V_{0}$ is a hypersurface in $\mathbb{P} V_{0}$ of degree 4 and it has to be $X_{1}$.

(b) $J_{4}=\left(0,0,0 ; x_{2}, x_{2}, x_{2}\right)$ has rank 3 so $p\left(0, J_{4}, 0,0\right) \in(X \backslash Y) \cap \mathbb{P} V_{0}$. Hence $Y \cap \mathbb{P} V_{0} \subseteq Y_{1} \cup Y_{2} . J_{5}=\left(0,0,0 ; x_{2}, 0,0\right)$ has rank 2 so $p\left(0, J_{5}, 0,0\right) \in$ $(Y \backslash Z) \cap\left(Y_{1} \backslash Z_{1}\right)$. Hence $Z \cap \mathbb{P} V_{0} \subseteq Y_{2}$ and $Y_{1} \subseteq Y \cap \mathbb{P} V_{0}$.

Let $v_{3}=\left(y_{2}, 0,0 \mid y_{3}, 0,0\right)$ so that $p v_{3} \in Y_{2} \backslash Z_{1}$. It is easy to check that $v_{3}$ satisfies $\partial f_{4} / \partial v=0$ in $\S 5.7$ so $p v_{2} \in Y$. This implies that $Y \supseteq Y_{2}$.

(c) We have seen in (b) that $Z \cap \mathbb{P} V_{0} \subseteq Y_{2} . \quad J_{6}=\left(0,0,0 ; y_{2}, 0,0\right)$ has rank 1 so $p\left(0, J_{6}, 0,0\right) \in Z \cap \mathbb{P} V_{0}$. Hence $Z \cap \mathbb{P} V_{0} \supseteq Z_{1}$. To complete the proof it suffices to show that $p v_{3} \notin Z$. Indeed otherwise, by Lemma 5.5.1, $v_{3}=p_{B}\left(0, J_{6}, 0,0\right)$ for some $B=\left(\beta_{i} ; d_{i}\right) \in \mathcal{J}$ (cf. (27)). Computations show that $p_{B}\left(J_{6}\right)=\left(0, J_{6}, 2 B \times J_{6}, 0\right)$. However $2 B \times J_{6}=\left(*, *, * ;-\beta_{1} y_{2}, *, *\right) \neq$ $\left(0,0,0 ; y_{3}, 0,0\right)$. Hence $p v_{3} \notin Z$.

Proof of Theorem 4.8.1. (a) This is determined by the map

$$
\begin{aligned}
r_{2} \quad: \quad \mathfrak{e}_{7} & =\mathbb{C} \otimes S^{2} U+V_{0,1}+U \otimes \pi_{\operatorname{Spin}_{12}}\left(\varpi_{6}\right) \rightarrow \\
& \rightarrow S^{2}(W \otimes U)=V_{2,0} \otimes S^{2} U+S^{2}(U)+V_{0,1} .
\end{aligned}
$$

The image of $r_{2}$ is nonzero so it is either $S^{2} U, V_{0,1}$ or the sum. By (42) (resp. (43)) $S^{2} U$ (resp. $V_{0,1}$ ) vanishes on $Y_{1}$ (resp. $Y_{2}$ ). By Lemma 9.3.1(c) the image cuts out $Z_{1}$ and hence it is must be the sum. Since $I^{\bullet}\left(Z_{1}\right)$ is generated by degree 2 polynomials, $R_{n}=A^{n}\left(Z_{1}\right)$.

(b)

$$
\begin{aligned}
r_{3}: V_{M} & =W \otimes U+\pi_{\operatorname{Spin}(12)}\left(\varpi_{5}\right) \rightarrow \\
& \rightarrow S^{3}\left(V_{0}\right)=V_{3,0} \otimes S^{3}(U)+W \otimes U+W \otimes S^{3}(U)+V_{1,2} \otimes U .
\end{aligned}
$$

Since $r_{3}$ is nontrivial, the image has to be $W \otimes U$ and they are the set of cubics in (40). We have shown that the set of cubics generates the ideal of $Y_{1} \cup Y_{2}$ and (b) follows from Theorem 3.5.1(b) and (44).

(c) By (20) and (41) we get

$$
\begin{array}{rl}
0 \rightarrow \operatorname{Res} \sigma_{X} \rightarrow \sum_{n, m} & \mathbf{H}\left(G^{\prime}, A^{m}\left(X_{1}\right)[27+m+4 n]\right) \\
\rightarrow \sum_{n, m} \mathbf{H}\left(G^{\prime}, A^{m}\left(X_{1}\right)[31+m+4 n]\right) \rightarrow 0 .
\end{array}
$$

The summands on the right also appear in the middle term. Therefore by removing these representations from (46) we get

$$
0 \rightarrow \operatorname{Res} \sigma_{X} \rightarrow \sum_{m=0}^{\infty} \mathbf{H}\left(G^{\prime}, A^{m}\left(X_{1}\right)[27+m+n]\right) \rightarrow 0 .
$$

This completes the Proof of Theorem 4.8.1(c). 
(d) This follows from Proposition 3.3.1 and (41).

9.4. Proof of Corollary 4.10.1. First we recall a well-known fact $[\mathbf{F H}]$.

Lemma 9.4.1. The 1 dimensional character $\operatorname{det}^{a-b}$ of $U_{2}$ is the only $S U_{2}$ fixed vector in $\pi_{S U_{3}}\left(a \varpi_{1}+b \varpi_{2}\right)$.

Consider the see-saw pair

$$
\begin{array}{lll}
E_{7,4} & & S U_{3} \\
& \triangleright \triangleleft & \\
E_{6,4} & & S U_{2}
\end{array}
$$

By Theorem 4.8.1(a), the trivial representation of $S U_{2}$ corresponds to the representation $\sigma_{Y}$ of $E_{7,4}$. Applying Lemma 9.4.1 to the see-saw pair (47) gives

(48)

$$
\begin{aligned}
& \sum_{a, b \geq 0} \Theta(a, b) \otimes \chi_{1}^{a-b} \\
& =\operatorname{Res}_{E_{6,4} \times U_{1}}^{E_{7,4}} \sigma_{Y} \\
& =\mathbf{H}\left(E_{6,4}, \mathbb{C}[12]\right) \otimes \chi_{1}^{0}+\sum_{a, b \geq 0} \sigma\left(E_{6,4}, \pi_{S U_{6}}\left(a \varpi_{1}+b \varpi_{5}\right)[a+b+10]\right) \otimes \chi_{1}^{a-b} .
\end{aligned}
$$

The second equality is Theorem 4.7.1(b). By Table 1B of the Appendix to [L3], $\Theta(a, b)$ contains the right-hand side of (17). Alternatively, one can deduce this by considering the correspondence of the infinitesimal characters [Li3]. By (48) the containment is an equality. This proves Corollary 4.10.1.

Acknowledgments. This paper is a continuation of the author's Ph.D thesis at Harvard $[\mathbf{L} \mathbf{1}]$ and $[\mathbf{L} 3]$. He thanks his thesis advisor Prof. Benedict Gross for introducing him to the subject of quaternionic representations. He also thanks the referee for his careful reading.

\section{References}

[Ba] W.L. Baily, Jr., An exceptional arithmetic group and its Eisenstein series, Ann. of Math., 91 (1970), 512-549, MR 42 \#4674, Zbl 0202.07901.

[Bou] Nicolas Bourbaki, Éléments de Mathematique: Groupes et Algèbres de Lie, Chapitres 4, 5 et 6, Hermann, 1968, MR 39 \#1590, Zbl 0186.33001.

[FH] W. Fulton and J. Harris, Representation Theory: A First Course, Graduate Text in Mathematics, 129, Springer-Verlag, 1991, MR 93a:20069, Zbl 0744.22001.

[GL] W.T. Gan and H.Y. Loke, Modular forms of level p on the exceptional tube domain, J. Ramanujan Math. Soc., 12(2) (1997), 161-202, MR 99c:11052, Zbl 0959.11019. 
[G] B. Gross, Letter to Kostant.

[GS] B. Gross and G. Savin, Motives with Galois group of type $G_{2}$ : An exceptional thetacorrespondence, Compositio Math., 114(2) (1998), 153-217, MR 2000e:11071, Zbl 0931.11015.

[GW1] B. Gross and N. Wallach, A distinguished family of unitary representations for the exceptional groups of real rank $=4$, in 'Lie Theory and Geometry: In Honor of Bertram Kostant,' Progress in Mathematics, 123, Birkhauser, Boston, 1994, MR 96i:22034, Zbl 0839.22006.

[GW2] _ On quaternionic discrete series representations and their continuations, J. Reine Angew. Math., 481 (1996), 73-123, MR 98f:22022, Zbl 0857.22012.

[HPS] J.S. Huang, P. Pandzic and G. Savin, New dual pair correspondences, Duke Math., 82(2) (1996), 447-471, MR 97c:22015, Zbl 0865.22009.

[J1] N. Jacobson, Exceptional Lie Algebras, Marcel Dekker, New York, 1971, MR 44 \#1707, Zbl 0215.38701.

[J2] _ Lie Algebras, Dover Publications Inc., New York, 1962, MR 26 \#1345, Zbl 0121.27504 .

[Kim] H. Kim, Exceptional modular form of weight 4 on an exceptional domain contained in $\mathbb{C}^{27}$, Revista Matematica Iberoamericana, 9(1) (1993), 139-200, MR 94c:11040, Zbl 0777.11015.

[Li1] J.-S. Li, Two reductive dual pairs in groups of type E, Manuscripta Math., 91 (1996), 163-177, MR 97j:22037, Zbl 0869.22008.

[Li2] _ On the discrete spectrum of $\left(G_{2}, P G S p_{6}\right)$, Invent. Math., 30 (1997), 189207, MR 98h:22014, Zbl 0913.22010.

[Li3] - The correspondences of infinitesimal characters for reductive dual pairs in simple Lie groups, Duke Math. J., 97(2) (1999), 347-377, MR 2000b:22014, Zbl 0949.22017.

[L1] H.Y. Loke, Exceptional Lie Algebras and Lie Groups, Part 2, Harvard Thesis, 1997.

[L2] - Dual pairs correspondences of $E_{8,4}$ and $E_{7,4}$. Israel J. Math., 113 (1999), 125-162, MR 2001k:22033, Zbl 0937.22009.

[L3] , Restrictions of quaternionic representations, J. Funct. Anal., 172 (2000), 377-403, MR 2001i:22017, Zbl 0953.22018.

[MS] K. Magaard and G. Savin, Exceptional $\Theta$-correspondences, I, Compositio Math., 107 (1997), 89-123, MR 98i:22015, Zbl 0878.22011.

[KP] W.G. McKay and J. Patera, Tables of Dimensions, Indices, and Branching Rules for Representations of Simple Lie Algebras, Lecture Notes in Pure and Applied Mathematics, 69, M. Dekker, 1981, MR 82i:17008, Zbl 0448.17001.

[SK] M. Sato and T. Kimura, A classification of irreducible prehomogeneous vector spaces and their relative invariants, Nagoya Math. J, 65 (1977), 1-155, MR 55 \#3341, Zbl 0321.14030.

[S1] W. Schmid, Homogeneous complex manifolds and representations of simple Lie groups, Dissertation, University of California, Berkeley, 1967; reprinted in 'Representation Theory and Harmonic Analysis on Semisimple Lie Groups', Mathematical Surveys and Monographs, 31, AMS, Providence, 1989, 223-286, MR 90i:22025, Zbl 0744.22016.

[W1] H.W. Wong, Dolbeault Cohomology Realization of Zuckerman Modules Associated with Finite Rank Representations, Dissertation, Harvard University, 1991. 
[W2] Dolbeault cohomology realization of Zuckerman modules associated with finite rank representations, J. Funct. Anal., 129 (1995), 428-454, MR 96c:22024, Zbl 0855.22014.

Received December 13, 1999 and revised February 9, 2000.

Department of Mathematics

National University of Songapore

10 Kent Ridge Crescent

SINGAPORE 119260

E-mail address: matlhy@math.nus.edu.sg 